\title{
Banque Centrale Et Politique Budgetaire : Une Évidence Empirique Pour La Zone CEMAC ${ }^{13}$
}

\author{
Tadadjeu Wemba Dessy-Karl, Doctorant \\ Kamajou François, Professeur Emérite \\ Noula Armand Gilbert, Maitre de conférence
}

Faculté des Sciences Economiques et de Gestion, Université de Dschang,

Département d'Analyse et de Politique Economique, Cameroun

Doi: 10.19044/esj.2018.v14n10p190 URL:http://dx.doi.org/10.19044/esj.2018.v14n10p190

\begin{abstract}
The purpose of this article is to analyze how the degree of independence of the Bank of Central African States (BEAC) influences the level of budget deficit for the countries of the Economic and Monetary Community of Africa Centrale (CEMAC) over the period 1994-2016. To do this, we rely on the statutory and rotational indicators of central bank governors developed by the literature, as well as on the econometrics of panel data. The results of the estimates indicate a mixed relationship between degree of independence and level of budget deficit. This result can be interpreted as an absence of constraint on fiscal policy, or as the partial assumption of cyclical stabilization by the central bank even when it is independent. It can also be interpreted as an absence of obligation for the budgetary policy to take charge of the cyclical adjustment. These results nevertheless confirm that the average orientation of fiscal policy is determined more by the budget procedure itself than by the statutes and the government of BEAC.
\end{abstract}

Keywords: Central bank independence, budget balance, CEMAC, BEAC, panel data

\section{Résumé}

L'objectif de cet article est d'analyser la manière dont le degré d'indépendance de la Banque des Etats de l'Afrique Centrale (BEAC) influence le niveau de déficit budgétaire pour les pays de la Communauté Economique et Monétaire de l'Afrique Centrale (CEMAC) sur la période 1994-2016. Pour cela, nous nous appuyons sur les indicateurs statutaires et de

${ }^{13}$ Cette étude reflète les idées personnelles des auteurs et n'exprime pas nécessairement la position de la Banque des Etats de l'Afrique Centrale (BEAC) ; bien entendu les éventuelles erreurs demeurent le seul fait des auteurs. 
rotation des gouverneurs de banques centrales développés par la littérature, ainsi que sur l'économétrie des données de panels. Les résultats des estimations indiquent une relation mitigée entre degré d'indépendance et niveau de déficit budgétaire. Ce résultat peut s'interpréter comme une absence de contrainte sur la politique budgétaire, ou comme la prise en charge partielle de la stabilisation conjoncturelle par la banque centrale même lorsque celle-ci est indépendante. Il peut aussi s'interpréter comme une absence d'obligation pour la politique budgétaire de prendre en charge le réglage conjoncturel. Ces résultats confirment néanmoins que l'orientation moyenne de la politique budgétaire est davantage déterminée par la procédure budgétaire elle-même que par les statuts et la gouvernement de la BEAC.

Mots clés : indépendance de la banque centrale, solde budgétaire, CEMAC, BEAC, données de panels

\section{Introduction}

Depuis l'affirmation de l'importance de l'indépendance ${ }^{14}$ des autorités monétaires vis-à-vis des autorités politiques, plusieurs travaux empiriques cherchent à renforcer cette nécessité en cherchant à établir quel est l'effet de cette indépendance sur les performances macroéconomiques (Bensafta, 2011). En général, il s'agit de trouver l'impact du degré d'indépendance des banques centrales sur l'inflation, la stabilité des prix, la croissance, la stabilité du produit et d'autres variables comme le déficit, la productivité, le chômage...etc.

Suivant la terminologie introduite par Debelle et Fischer (1994), l'Indépendance d'une Banque Centrale (IBC) peut être subdivisée en deux catégories : indépendance d'objectif et indépendance d'instrument. Cette distinction reflète toutefois celle établie par Grilli et al. (1991) entre indépendance politique et indépendance économique. Par indépendance d'objectifs, Debelle et Fischer (1994) entendent l'autonomie dont dispose la banque centrale dans le choix des objectifs finals de politique monétaire. L'indépendance opérationnelle se réfère quant à elle à la liberté effective dont dispose l'autorité monétaire dans le choix et le maniement de ses instruments pour atteindre les objectifs qui lui ont été assignés. En conséquence, elle apparaît pour une banque centrale comme un gage fort dans la conquête de la crédibilité basée sur l'engagement anti inflationniste des autorités monétaires (Avom et Bobbo, 2013).

${ }^{14}$ Le corpus théorique qui accompagne cet argument, ou plus précisément qui propose la solution de la banque centrale indépendante, résulte des travaux menés dès le début des années soixante-dix autours d'une problématique très actuelle en théorie monétaire, celle de l'incohérence temporelle. Le problème de l'incohérence temporelle est présenté pour la première fois dans un article publié par Kydland et Prescott (1977). 
Depuis les années quatre-vingt, la plupart des analyses à partir d'indicateurs quantifiés ont mis l'accent sur l'effet d'une telle indépendance sur l'inflation et son compromis potentiel avec la croissance économique (Grilli et al. (1991), Cukierman et al. (1992), Franzese (1999, 2002), Keefer et Stasavage (2003), Crowe et Meade (2008)). Néanmoins, certains auteurs ont pu montrer que les statuts de la banque centrale peuvent affecter la conduite de la politique budgétaire par deux canaux principaux. En premier lieu, l'indépendance de la banque centrale interdit d'avoir recours à la planche à billets ou à un quelconque accès privilégié au crédit pour financer un déficit des comptes publics. Elle ne permet pas non plus de dévaloriser la dette publique par l'inflation. Elle incite donc le pouvoir budgétaire à davantage de discipline. En second lieu, la spécialisation de la politique monétaire dans la lutte contre l'inflation implique que la politique budgétaire se charge de la stabilisation. Il peut arriver que l'indépendance de la banque centrale et sa spécialisation dans la lutte contre l'inflation conduisent les autorités publiques à faire un usage conjoncturel accru de l'instrument budgétaire ${ }^{15}$. La situation de la France de 1993 à 1994 peut illustrer en partie cette logique conjoncturelle (Bénassy-Quéré et Pisani-Ferry, 1994).

En Afrique, les besoins en seigneuriage ont tendance à augmenter avec des déficits budgétaires des Etats. Dans la Communauté Economique et Monétaire de l'Afrique Centrale (CEMAC) ${ }^{16}$, la politique monétaire échappe au pouvoir discrétionnaire des Etats et est conduite par un institut d'émission indépendant : la Banque des Etats de l'Afrique Centrale (BEAC) ${ }^{17}$.

Déjà jusqu'au début des années 2000, très peu d'études empiriques ont portés sur la relation entre l'IBC et la politique budgétaire. La plupart d'entre elles se sont concentrées sur les pays développées et ont données des résultats mixtes et peu concluants (Neyapti, 2003).

En effet, comme le montre l'enquête réalisée par Batini et Laxton (2006) auprès d'un grand nombre de banques centrales, l'adoption du ciblage d'inflation au sein des économies émergentes et en voies de développement s'est caractérisée par une profonde réforme des statuts de la banque centrale,

${ }^{15}$ Les banques centrales indépendantes sont favorables à la discipline budgétaire à cause de la relation de long terme qui existe entre les déficits et l'inflation. Ainsi, elles peuvent poursuivre leurs préférences en matière de politique budgétaire en raison de la hausse des taux d'intérêt et du refus de prêter au gouvernement.

${ }^{16}$ La CEMAC est constituée de six (06) pays à savoir : le Cameroun, le Congo, le Gabon, la Guinée Equatoriale, la République Centrafricaine et le Tchad.

${ }^{17}$ Afin de renforcer la gouvernance de la BEAC, le Comité ministériel de l'Union Monétaire de l'Afrique Centrale (UMAC) après avis conforme du Conseil d'administration de la Banque Centrale a adopté successivement deux réformes des statuts en Septembre 2007 et en Octobre 2010. Ces statuts réaffirment l'indépendance de la BEAC, en interdisant que lui soit imposé toute obligation ou contrôle autres que ceux définis dans ses statuts ou dans la convention de l'UMAC. 
visant notamment à limiter voire interdire le financement monétaire du déficit public et accroitre son indépendance. Or comme le précise Lucotte (2012), les recettes de seigneuriage représentant une part importante des ressources publiques au sein de ces pays et les autorités budgétaires concernées sont généralement incitées à compenser cette perte de revenu par une source de financement alternative. Partant de là, la préoccupation serait alors de savoir quel est l'impact du degré d'indépendance d'une banque centrale sur la conduite de la politique budgétaire ? A travers cette interrogation ramenée à la zone $\mathrm{CEMAC}^{18}$, l'objectif de ce papier est d'évaluer l'importance de l'indépendance de la BEAC sur la discipline budgétaire ${ }^{19}$ dans cette Zone.

Cette étude apporte une contribution importante à la recherche existante à ce sujet sur deux points. Premièrement, elle fournit un cadre théorique unifiant qui relie l'IBC à la performance budgétaire et étend les tests empiriques de cette relation au-delà de la recherche dans les pays développés. Deuxièmement, nous cherchons à combler certaines lacunes dans la littérature et plus précisément en zone Franc CFA. Le reste de la rédaction sera dans l'ordre des sections suivantes : littérature, méthodologie, résultats et enfin conclusion.

\section{Revue de la littérature : politique monétaire et politique budgétaire une double causalité}

A la suite de l'étude pionnière de Sargent et Wallace (1981), une vaste littérature théorique s'est attachée à analyser les interactions entre politiques monétaire et budgétaire ${ }^{20}$. Cette littérature riche et complexe peut être divisée en deux courants de recherche distincts. Le premier a montré le rôle prépondérant de la politique budgétaire dans la formulation de la politique monétaire, tandis que le second a quant à lui mis en évidence la relation inverse. Nous présentons successivement ces deux champs de la littérature théorique.

Dans un article désormais devenu célèbre, Sargent et Wallace (1981) prennent le contre-pied des thèses monétaristes en affirmant que l'inflation n'est pas simplement un phénomène monétaire, mais qu'elle peut également trouver son origine dans la conduite de la politique budgétaire. Considérant une structure de jeu non coopératif de type " poule mouillée », Sargent et

\footnotetext{
${ }^{18}$ Voir les nouveaux critères de surveillance multilatérale de la CEMAC.

${ }^{19}$ Plusieurs indicateurs peuvent être retenus pour apprécier le degré de discipline budgétaire des Etats. Le plus immédiat est le solde financier des administrations publiques rapporté au PIB en valeur. C'est aussi l'indicateur le plus usuel et le plus fréquemment commenté. Le second est la dette publique et troisième est le solde primaire, c'est-à-dire le solde financier hors intérêts de la dette publique.

${ }^{20}$ Pour une description simplifiée des différents scénarii possibles entre politiques monétaire et budgétaire modélisables à l'aide de la théorie des jeux, le lecteur se référera au récent article de Franta et al. (2011).
} 
Wallace (1981) montrent en effet qu'une politique budgétaire exagérément laxiste peut contraindre la politique monétaire à se détourner de son objectif de stabilité des prix et à monétiser la dette publique, de manière à éviter que cette dernière ne prenne une trajectoire explosive. Dans les termes de Sargent et Wallace (1981), le gouvernement est alors en position de « dominance budgétaire », en ce sens que la banque centrale n'a pas d'autre choix que de s'ajuster au comportement laxiste de l'autorité budgétaire afin de satisfaire la contrainte budgétaire de cette dernière ${ }^{21}$. Quand bien même la banque centrale souhaiterait se montrer plus rigoureuse en choisissant un niveau de seigneuriage plus faible, cela se traduira par une augmentation durable de la dette publique et donc, du seigneuriage qu'il sera nécessaire de dégager dans le futur pour stabiliser cette dernière. Sargent et Wallace (1981) montrent ainsi qu'entre une autorité monétaire rigoureuse et une autorité budgétaire laxiste, c'est l'autorité monétaire qui finira toujours par céder en se détournant de son objectif de stabilité des prix.

Ce schéma, qualifié par Sargent et Wallace (1981) «d'arithmétique monétariste déplaisante », a connu un large écho dans la littérature théorique. Dans une série de contributions récentes en réponse à la forte hausse des dettes souveraines d'après-crise, Leeper et Walker (2011) et Davig et al. (2011) montrent par exemple que lorsque le taux d'endettement public a atteint ses limites et qu'aucun ajustement budgétaire n'est possible, la politique monétaire n'a pas d'autre choix que de monétiser la dette publique et de se détourner de son objectif de stabilité des prix afin de stabiliser la dette publique. La politique monétaire est alors qualifiée de « passive » par Leeper et Walker (2011) et Davig et al. (2011). Cette incitation à monétiser la dette publique sera d'autant plus forte que la part de la dette non indexée est élevée (Calvo, 1989). La conséquence directe est alors une élévation permanente de l'inflation à long terme. En outre, comme le souligne Villieu (2011), même sous l'hypothèse d'une autorité monétaire "dominante », des ratios élevés d'endettement public peuvent conduire à un accroissement de l'inflation à court terme si une monétisation future de la dette est anticipée. Les résultats de la littérature empirique ayant étudié le lien entre politique budgétaire et inflation sont toutefois relativement mitigés ${ }^{22}$.

En marge de ces différents travaux, un courant de pensées va plus loin et montre que le niveau d'inflation est indépendant de l'orientation de la politique monétaire (restrictive ou accommodante) et ne dépend que de la conduite de la politique budgétaire du gouvernement. Ce courant, initié notamment par Woodford (1994, 1995) et Sims (1994), est plus

21 Voir notamment Christiano et Fitzgerald (2000) pour une présentation simplifiée du phénomène de « dominance budgétaire ».

${ }^{22}$ Voir notamment Catão et Terrones (2005) pour une synthèse des travaux empiriques relatifs à la question du lien entre politique budgétaire (dette et déficit publics) et inflation. 
communément connu sous le nom de «théorie fiscale des prix ». Se plaçant dans un cadre de politique budgétaire non ricardienne, c'est-à-dire ne se souciant pas de sa contrainte budgétaire inter temporelle, ces auteurs montrent en effet que le niveau général des prix qui s'établit est le seul qui assure l'équilibre de cette contrainte. Pour Woodford (1996) et Beetsma et Bovenberg (1999), la seule façon de limiter la volatilité des prix est alors de définir des règles budgétaires et des plafonds d'endettement garantissant l'équilibre des finances publiques et la soutenabilité de la dette publique, permettant ainsi d'immuniser la banque centrale des pressions inflationnistes du gouvernement. La théorie fiscale des prix a toutefois reçu un accueil très mitigé, et nombre d'auteurs se sont attachés à en montrer les limites (Cushing (1999) et Mc Callum (2001)).

Parallèlement à ces divers travaux qui ont mis en avant les conséquences néfastes d'une politique budgétaire laxiste sur la crédibilité de la politique monétaire et souligné la nécessité d'une coordination étroite entre ces deux politiques, un second courant de recherche a quant à lui cherché à étudier la causalité inverse, c'est-à-dire l'impact de la politique monétaire sur la conduite de la politique budgétaire et fiscale. L'un des papiers théoriques fondateurs de ce courant est l'article de Tabellini (1986), approfondi par la suite par Alesina et Tabellini (1987). Dans le cadre d'un modèle statique à un pays, ces auteurs considèrent une autorité monétaire et une autorité budgétaire indépendantes, la première contrôlant le taux d'inflation, tandis que la seconde choisit le taux d'imposition lui permettant de financer les dépenses publiques. La question de l'endettement public n'est pas prise en compte dans le modèle et les deux sources de financement des dépenses publiques sont les recettes fiscales et les recettes de seigneuriage. En outre, la banque centrale et le gouvernement minimisent une fonction de perte qui dépend du revenu, de l'inflation et des dépenses publiques, mais dont les coefficients de pondération attachés à chacun de ces objectifs sont différents, reflétant une divergence dans les objectifs de ces deux autorités. Partant de ces hypothèses, Alesina et Tabellini (1987) montrent alors qu'un resserrement de la politique monétaire, dont la conséquence directe est une baisse des recettes de seigneuriage pour le gouvernement, va inciter ce dernier à accroître la pression fiscale afin de satisfaire sa contrainte budgétaire.

Ce courant de la littérature a par la suite connu une production relativement foisonnante, avec des travaux prenant des directions de recherche complémentaires. Certains de ces travaux ont tout d'abord cherché à approfondir les résultats d'Alesina et Tabellini (1987) en introduisant de la dynamique dans le modèle afin de prendre en compte la question de la dette publique (Van Arle et al. (1995)). D'autres travaux ont quant à eux élargi le cadre d'analyse en étudiant les interactions entre politique monétaire et politique budgétaire dans le cadre d'une union monétaire. Parmi ces travaux, 
on retrouve notamment une série de contributions de Beetsma et Bovenberg (1997, 1998). Le modèle développé par Beetsma et Bovenberg (1998) montre en particulier, qu'en l'absence de coopération budgétaire, la conduite d'une politique monétaire conservatrice au sein d'une union monétaire peut inciter les gouvernements à diminuer leurs dépenses publiques, réduisant de ce fait le biais de dépenses publiques.

Plus récemment, modifiant la séquentialité du modèle développé par Huang et Wei (2006), Minea et al. (2012) ont pour leur part cherché à étudier dans quelle mesure le niveau de la cible d'inflation poursuivi par une banque centrale pouvait impacter l'effort de mobilisation fiscale du gouvernement. Conformément aux travaux précédemment cités mettant en évidence un possible impact de la politique monétaire sur la conduite de la politique budgétaire, les résultats de leur modèle suggèrent une relation négative entre le niveau de la cible d'inflation et l'effort de mobilisation fiscale du gouvernement. Plus précisément, selon ces auteurs, l'incitation d'un gouvernement à réformer son administration fiscale serait d'autant plus forte que la cible d'inflation est basse, du fait d'une baisse significative des recettes de seigneuriage.

\section{Indépendance de la banque centrale et discipline budgétaire : des résultats empiriques controversés}

Dans la pratique, la probabilité qu'une autorité monétaire ne cède pas aux pressions du gouvernement est d'autant plus grande que cette autorité est indépendante du gouvernement. Il existerait dès lors une relation positive (négative) entre indépendance de la banque centrale et discipline (laxisme) budgétaire. Partant de cela, un certain nombre d'études ont cherché à tester empiriquement l'existence d'un tel lien. Ces études se sont toutefois majoritairement focalisées sur les économies industrialisées.

La première de ces études est celle conduite par Burdekin et Laney (1988) portant sur un échantillon de 12 pays de l'OCDE pour la période 19601983. Ces auteurs retiennent le déficit public (en \% du PIB) comme variable endogène et mesurent l'indépendance de la banque centrale à l'aide d'une variable binaire, cette dernière prenant la valeur 1 si l'autorité monétaire est jugée comme indépendante et 0 autrement. Les autorités monétaires considérées comme indépendantes sont ici les banques centrales du Canada, des Etats-Unis, de Suisse et d'Allemagne de l'Ouest. Les résultats obtenus par Burdekin et Laney (1988) révèlent une relation négative et significative entre l'indépendance de la banque centrale et le niveau de déficit public. Ces résultats vont dans le même sens que l'étude de cas réalisée par Masciandaro et Tabellini (1988), qui comparent les niveaux de déficit public de l'Australie, du Canada, du Japon, de la Nouvelle-Zélande et des Etats-Unis sur la période 1970-1985. Les statistiques indiquent que la Nouvelle-Zélande, dont la banque 
centrale est considérée comme peu indépendante du gouvernement, est effectivement l'économie caractérisée par le déficit public le plus élevé.

Des articles ultérieurs ont cherché à prolonger l'étude de Burdekin et Laney (1988) en s'appuyant notamment sur des indicateurs quantitatifs d'indépendance de la banque centrale récemment développés dans la littérature $^{23}$. Parmi ces études, on retrouve celle de Grilli et al. (1991), qui développent un indicateur d'indépendance légale de la banque centrale (1'IBCGMT), distinguant indépendance économique et indépendance politique. S'appuyant sur un échantillon de 18 pays de l'OCDE pour la période 19501989, ces auteurs ne trouvent toutefois pas de relation statistiquement significative entre le degré d'indépendance de l'autorité monétaire et le déficit budgétaire. Des résultats similaires sont trouvés par Pollard (1993) à partir d'une analyse statistique portant sur 16 pays de l'OCDE pour la période 19731989 à l'aide de l'indicateur d'indépendance développé par Alesina et Summers (1993).

Suite à la publication par Cukierman et al. (1992) et Eijffinger et Schaling (1992) de deux nouveaux indicateurs d'indépendance, De Haan et Sturm (1992) ont ré-estimé la relation entre indépendance de la banque centrale et niveau de déficit public pour un échantillon de 18 pays de l'OCDE couvrant la période 1961-1987. Pour cela, les auteurs s'appuient successivement sur les quatre indicateurs d'indépendance précédemment cités. Les résultats indiquent un lien négatif entre indépendance de la banque centrale et déficit budgétaire, même si la significativité statistique de la relation varie suivant l'indicateur d'indépendance retenu et les sous-périodes considérés. Cette sensibilité des résultats à l'indicateur d'indépendance employé est confirmée par l'étude statistique menée par Bénassy-Quéré et Pisany-Ferry (1994) portant sur 20 pays de l'OCDE entre 1978 et 1992. Afin de mesurer le degré de discipline budgétaire des gouvernements, ces auteurs retiennent trois indicateurs : le déficit public, la dette publique et le solde primaire, ces trois variables étant exprimées en pourcentage du PIB. Les résultats révèlent un lien beaucoup plus ténu entre le degré d'indépendance de l'autorité monétaire et la discipline budgétaire lorsque l'autonomie de la banque centrale est mesurée à l'aide de l'indicateur de Grilli et al. (1991), que lorsqu'elle est mesurée à l'aide de l'indicateur Cukierman et al. (1992).

En outre, comme le soulignent Grilli et al. (1991), nous avons pu observer dans la plupart des pays de l'OCDE un déclin des recettes de seigneuriage à partir des années 1980, alors même que les déficits budgétaires étaient relativement élevés. Dès lors, il semble que l'étude du lien entre indépendance de la banque centrale et discipline budgétaire soit plus

${ }^{23}$ Voir notamment Eijffinger et De Haan (1996) et Banain (2008) pour une description détaillée des différents indicateurs d'indépendance de la banque centrale développés dans la littérature. 
pertinente dans le cadre des économies émergentes et en développement. Pour ces dernières, le renforcement statutaire de l'indépendance de l'autorité monétaire, traditionnellement accompagné par des mesures visant à restreindre les avances de la banque centrale au Trésor, se traduira en effet par une baisse importante des recettes de seigneuriage, forçant ainsi le gouvernement à davantage de prudence dans la conduite de sa politique budgétaire.

Deux papiers empiriques se sont attachés à tester l'existence d'un lien entre indépendance de la banque centrale et déficit budgétaire au sein des pays émergents et en développement. Le premier est celui de Sikken et De Haan (1998) conduit sur un échantillon de 30 pays en développement pour la période 1972-1989. Les auteurs s'appuient sur deux mesures complémentaires de l'indépendance : l'indicateur agrégé et désagrégé de Cukierman et al. (1992) et, le taux de rotation des gouverneurs (le TOR) (Cukierman et al., 1992), qui est un proxy inverse de l'indépendance réelle de la banque centrale. Les résultats des estimations en coupe transversale conduites par Sikken et De Haan (1998) indiquent une relation non significative entre le degré d'indépendance et le niveau de déficit public et ce, quel que soit l'indicateur d'indépendance utilisé. Plus récemment, s'appuyant sur les mêmes indicateurs d'indépendance, Lucotte (2009) ré-estime cette relation à l'aide d'un modèle à effets aléatoires appliqué à un panel de 78 pays en développement de 1995 à 2004. Les résultats obtenus à l'aide du taux de rotation des gouverneurs confirment l'effet disciplinant de l'indépendance de la banque centrale sur les finances publiques, effet qui est non significatif lorsque c'est l'indicateur d'indépendance légale qui est considéré. Ce résultat est toutefois cohérent avec l'argument de Cukierman et al. (1992), qui montrent que pour la majeure partie des pays émergents et en développement, l'indépendance légale n'est pas une mesure pertinente du degré d'autonomie effective des banques centrales.

Enfin Bodea (2013) et, Bodea et Higashijima (2017) rapportent pour un échantillon de 23 pays démocratiques et non-démocratiques postcommunistes (d'Europe de l'Est, d'Amérique latine et des Caraïbes) que l'indépendance de ces banques centrales limite les déficits budgétaires et a un effet dissuasif sur les dépenses excessives des gouvernements sur la période 1970-2007.

Le tableau 1 ci-dessous fait un récapitulatif des principales études empiriques menées sur cette relation entre l'indépendance de la banque centrale et la discipline budgétaire. 
Tableau 1 : Principales études empiriques sur la relation entre l'indépendance de la banque centrale et les déficits budgétaires

\begin{tabular}{|c|c|c|c|c|}
\hline $\begin{array}{l}\text { Auteurs et } \\
\text { années }\end{array}$ & $\begin{array}{c}\text { Pays ou groupes de } \\
\text { pays }\end{array}$ & $\begin{array}{l}\text { Période } \\
\text { d'étude }\end{array}$ & $\begin{array}{l}\text { Indice d'indépendance } \\
\text { de la banque centrale }\end{array}$ & $\begin{array}{l}\text { Résultats de la } \\
\text { relation entre } \\
\text { IBC et déficits } \\
\text { budgétaires }\end{array}$ \\
\hline $\begin{array}{l}\text { Masciandaro et } \\
\text { Tabellini (1987) }\end{array}$ & $\begin{array}{c}5 \text { pays } \\
\text { industrialisés }\end{array}$ & $1970-1985$ & Indice-GMT & $\begin{array}{l}\text { Négatif et non } \\
\text { significatif. }\end{array}$ \\
\hline $\begin{array}{c}\text { Burdekin et } \\
\text { Laney (1988) }\end{array}$ & $\begin{array}{l}12 \text { pays de } \\
\text { l'OCDE }\end{array}$ & 1960-1983 & Variables Dummy & $\begin{array}{l}\text { Négatif et } \\
\text { significatif. }\end{array}$ \\
\hline $\begin{array}{l}\text { Grilli et al. } \\
\text { (1991) }\end{array}$ & $\begin{array}{l}18 \text { pays de } \\
\text { l'OCDE }\end{array}$ & $1950-1989$ & Indice-GMT & $\begin{array}{l}\text { Négatifs et non } \\
\text { significatif. }\end{array}$ \\
\hline $\begin{array}{c}\text { De Haan et } \\
\text { Sturm (1992) }\end{array}$ & $\begin{array}{l}18 \text { pays de } \\
\text { l'OCDE }\end{array}$ & 1961-1987 & $\begin{array}{c}5 \text { indices différents } \\
\text { d'IBC }\end{array}$ & $\begin{array}{l}\text { Négatif et } \\
\text { significatif }\end{array}$ \\
\hline Pollard (1993) & $\begin{array}{l}16 \text { pays de } \\
\text { l'OCDE }\end{array}$ & 1973-1989 & $\begin{array}{l}\text { Indices d'Alesina et } \\
\text { Summers (1993) }\end{array}$ & $\begin{array}{l}\text { Négatif et non } \\
\text { significatif. }\end{array}$ \\
\hline $\begin{array}{c}\text { Bénassy-Quéré } \\
\text { et Pisani-Ferry } \\
\text { (1994) }\end{array}$ & $\begin{array}{l}20 \text { pays de } \\
\text { l'OCDE }\end{array}$ & 1978-1992 & $\begin{array}{c}\text { Indice-CWN et indice- } \\
\text { GMT }\end{array}$ & $\begin{array}{l}\text { Négatif et non } \\
\text { significatif. }\end{array}$ \\
\hline $\begin{array}{l}\text { Sikken et De } \\
\text { Haan (1998) }\end{array}$ & 30 pays développés & 1972-1989 & Indice-CWN & $\begin{array}{c}\text { Non significatif } \\
\text { et ambiguïté des } \\
\text { signes. }\end{array}$ \\
\hline $\begin{array}{l}\text { D'Amato et al. } \\
\text { (2009) }\end{array}$ & $\begin{array}{l}55 \text { pays dont } \\
23 \text { de l'OCDE et } \\
32 \text { non-OCDE }\end{array}$ & 1980-1989 & Indice-CWN & $\begin{array}{l}\text { Négatif et non } \\
\text { significatif- } \\
\text { OCDE ; Négatif } \\
\text { et significatif } \\
\text { non-OCDE. }\end{array}$ \\
\hline Lucotte (2009) & $\begin{array}{l}78 \text { pays sous- } \\
\text { développés }\end{array}$ & $1995-2004$ & Indice-CWN et TOR & $\begin{array}{c}\text { TOR négatif et } \\
\text { significatif ; } \\
\text { CWN négatif } \\
\text { non significatif. }\end{array}$ \\
\hline $\begin{array}{l}\text { Mpofu Sehliselo } \\
\text { (2012) }\end{array}$ & $\begin{array}{l}20 \text { pays d'Afrique } \\
\text { Australe, du Nord } \\
\text { et du Sud } \\
\text { n'appartenant pas à } \\
\text { des unions } \\
\text { monétaires }\end{array}$ & 1990-2008 & Indice-CWN et TOR & $\begin{array}{l}\text { Indice TOR et } \\
\text { CWN positifs et } \\
\text { non significatifs. }\end{array}$ \\
\hline $\begin{array}{l}\text { Buderkin et } \\
\text { Laney } \\
(2015)\end{array}$ & $\begin{array}{c}14 \text { pays } \\
\text { d'Amérique Latine }\end{array}$ & 1990-2012 & Indice-CWN & $\begin{array}{l}\text { Négatif et } \\
\text { significatif. }\end{array}$ \\
\hline $\begin{array}{c}\text { Bodea (2013) } \\
\text { Bodea et } \\
\text { Higashijima } \\
(2017)\end{array}$ & $\begin{array}{c}23 \text { pays } \\
\text { démocratiques et } \\
\text { non démocratiques }\end{array}$ & $1970-2007$ & Indice-CWN & $\begin{array}{l}\text { Négatif et } \\
\text { significatif. }\end{array}$ \\
\hline
\end{tabular}

\section{Source : Construction des auteurs.}

Avec : Indice-GMT : Indice d'indépendance légale de Grilli et al (1991); Indice-CWN : Indice d'indépendance légale de Cukierman et al. (1992); TOR : Indice d'indépendance réelle de Cukierman et al. (1992) / taux de rotation des gouverneurs. 


\section{Méthodologie}

\section{Nature et source des données}

Les données recueillies pour cette étude sont de sources secondaires. Elles proviennent de diverses sources telles que : les bases statistiques de la Banque Mondiale dans le «World Development Indicators (2016) et des rapports annuels d'activités du Fonds Monétaire International, de la Banque de France, et de la BEAC.

Les données institutionnelles telles que le degré d'indépendance légale et réelle de la BEAC sur la période 2010-2016 ont été calculé par nos propres soins à partir de la méthodologie de Cukierman et al. (1992). Celles sur la période 1994-2009 ont été extraite de la base de données de Crowe et Meade (2008) du FMI Central Bank Laws Database et Morgan Stanley's Central Bank Directory (1995-2005).

Nos données sont de nature quantitative. L'étude porte sur les six (06) pays de la CEMAC (le Cameroun, le Congo, le Gabon, la Guinée Equatoriale, la République Centrafricaine et le Tchad) dont les spécificités peuvent être communes ou différentes. La périodicité est annuelle et part de 1994 à $2016^{24}$.

\section{Mesure légale et réelle de l'indépendance de la BEAC}

A l'instar de Mpofu (2012), Ngniado (2016) et Garriga (2016, 2017), nous utilisons les indices de Cukierman et al. (1992) car ils sont le plus adaptés au pays en voie de développement. En effet, Cukierman, Webb et Neyapti (CWN) (1992) ont développé deux (02) indices d'indépendance de la banque centrale : un indice légal ${ }^{25}$ (LIBC-CWN) et un indice réel à savoir le taux de rotation ${ }^{26}$ des gouverneurs des banques centrales (TOR). Nous avons choisi cet indice sur la base de sa large popularité dans la littérature et l'analyse empirique, la disponibilité des données, la cohérence des questionnaires et formules, la facilité de calcul et les biais de subjectivité limitées.

\section{$\checkmark$ Indépendance légale de la BEAC}

Pour apprécier le degré d'indépendance légale de la BEAC, nous avons eu recours dans le tableau de l'annexe 3 comme Bessma Momani et St. Armant (2014) à la méthodologie de Garriga (2016 et 2017), inspirée de l'étude initiale menée par Cukierman, Webb et Neyapti (CWN) (1992). D'après nos calculs, l'indice d'indépendance légale (LIBC-CWN) de la BEAC

\footnotetext{
${ }^{24}$ Nous ne séparons pas la période d'étude en deux sous périodes c'est-à-dire, 1994-2009 avant les réformes statutaires de la BEAC et 2010-2016 après les réformes car, il y n'a pas une grande variation des indices d'indépendance de la banque centrale avant et après ces réformes.

${ }^{25}$ Plus cette valeur se rapproche de 1 , plus la banque centrale sera dite indépendante sur le plan légal.

${ }^{26}$ Les résultats du TOR vont de 0 point (niveau le plus élevé d'indépendance réelle) à 1 point (niveau le plus bas d'indépendance réelle).
} 
entre 2010 et 2016 est de $\mathbf{0 . 6 0}$ point ; ce qui fait d'elle une banque centrale indépendante sur le plan légal. Nous remarquons que cet indice est resté statique au fil des années et plus précisément après les réformes de 2010. De 1994 à 2009, il était également de $\mathbf{0 . 6 0}$ point (Crowe et Meade, 2008). L'analyse de Ngniado (2016) a montré que cet indice était de $\mathbf{0 . 5 8}$ point entre 2010 et 2015 , ce qui n'est pas loin du nôtre. On peut évoquer ainsi une certaine portée limitée des réformes statutaires de la BEAC.

\section{$\checkmark$ Indépendance réelle de la BEAC}

Afin de mesurer le degré d'indépendance réelle (TOR) de la BEAC sur la période 1994-2016, nous avons eu recours comme Mpofu (2012) à la méthode de Cukierman et al. (1992)

Nous constatons d'après le tableau de l'annexe 1 que notre TOR est de $\mathbf{0 . 1 3 0 4}$ point, soit 3 changements en 23 années. Cela fait de la BEAC une banque centrale indépendante sur le plan réel. Mais, il faut cependant remarquer qu'un taux de rotation faible ne signifie pas forcément que la banque centrale soit vraiment indépendante, car le gouverneur en place peut simplement y rester parce qu'il n'entre pas en conflit avec les autorités politiques (Mhamdi Ghrissi, 2009).

\section{Spécification des modèles et des variables Spécification des modèles}

Partant du modèle original proposé par Barro (1974), nous pouvons spécifier les nôtres (modèle $\mathbf{1}$ et $\mathbf{2}$ ) afin de déterminer l'effet de l'indépendance de la banque centrale sur la discipline budgétaire. Ainsi, deux (02) modèles économétriques sont à spécifier à savoir : le modèle 1 qui correspond à l'équation budgétaire en relation avec l'indépendance légale de la banque centrale (LIBC). Il se présente comme suit :

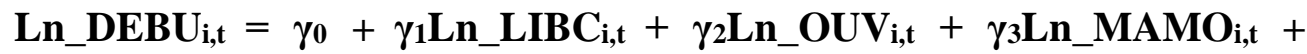

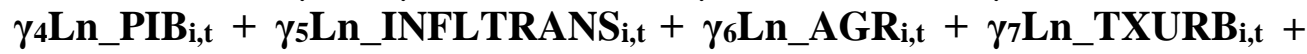
$\mathbf{Q}_{\mathbf{i}}+\mathbf{Y}_{\mathbf{t}}+\mathbf{Z}_{\mathbf{i t}}$

Et le modèle 2 qui correspond à l'équation budgétaire en relation avec l'indépendance réelle de la banque centrale (TOR). Il se présente comme suit:
Ln_DEBU $_{i, t}=\delta_{0}+\delta_{1} L_{2} \_T O R_{i, t}+\delta_{2} L_{2} \_O U V_{i, t}+\delta_{3} L_{-}$MAMO $_{i, t}+$

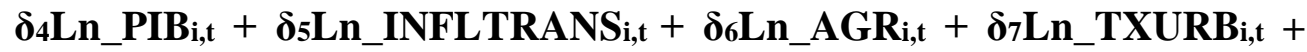 $\mathbf{U}_{\mathbf{i}}+\mathbf{V}_{\mathbf{t}}+\mathbf{W}_{\text {it }}$

Avec : Ln : l'opérateur logarithme ; $\mathbf{D E B} \mathbf{U}_{\mathbf{i}, \mathrm{t}}$ : le solde budgétaire en rapporté au PIB dans le pays $\mathbf{i}$ au cours de l'année $\mathbf{t}$ (dans notre analyse on 
parlera plutôt de déficit budgétaire ${ }^{27}$ ); $\mathbf{L I B C}_{\mathbf{i}, \mathbf{t}}$ : l'indice d'indépendance légale de la banque centrale (dont la valeur est comprise entre 0 et 1 ) dans le pays $\mathbf{i}$ au cours de l'année $\mathbf{t}$; $\mathbf{T O R}_{\mathbf{i}, \mathbf{t}}$ : l'indice de taux de rotation des gouverneurs de la banque centrale (dont la valeur est comprises entre 0 et 1) dans le pays $\mathbf{i}$ au cours de l'année $\mathbf{t}$; $\mathbf{O U V} \mathbf{i , t}$ : l'ouverture commerciale (somme des exportations et des importations rapporté au PIB) dans le pays $\mathbf{i}$ au cours de l'année $\mathbf{t}$; MAMO $_{\mathbf{i}, \mathbf{t}}$ : la monnaie et quasi monnaie (M2) en pourcentage de PIB dans le pays $\mathbf{i}$ au cours de l'année $\mathbf{t} ; \mathbf{P I B}_{\mathbf{i}, \mathbf{t}}$ : le taux de croissance réelle du produit intérieur brut par habitant dans le pays $\mathbf{i}$ au cours de l'année $\mathbf{t}$; TINFLATRANS $\mathbf{i , t}$ : le taux d'inflation (indice de prix à la consommation) transformé dans le pays $\mathbf{i}$ au cours de l'année $\mathbf{t} ; \mathbf{A G R}_{\mathbf{i}, \mathbf{t}}$ : la part de l'agriculture dans le PIB dans le pays $\mathbf{i}$ au cours de l'année $\mathbf{t}$; TXURB $_{\mathbf{i}, \mathbf{t}}$ : le taux d'urbanisation en pourcentage du PIB dans le pays $\mathbf{i}$ au cours de l'année $\mathbf{t} ; \mathbf{U}_{\mathbf{i}}$ et $\mathbf{Q}_{\mathbf{i}}$ : l'effet individu; $\mathbf{V}_{\mathbf{t}}$ et $\mathbf{Y}_{\mathbf{t}}$ : l'effet temps; $\mathbf{W}_{\mathbf{i t}}$ et $\mathbf{Z}_{\mathbf{i t}}$ : l'effet croisé ; $\mathbf{i}$ : nombre de pays allant de 1 à $6 ; \mathbf{t}:$ la période allant de 1994 à $2016 ; \boldsymbol{\gamma}_{\mathbf{0}}$ et $\boldsymbol{\delta}_{\mathbf{0}}:$ les constantes; $\boldsymbol{\gamma}_{\mathbf{1}}$ à $\boldsymbol{\gamma}_{\mathbf{7}}$ et $\boldsymbol{\delta}_{\mathbf{1}}$ à $\boldsymbol{\delta}_{\mathbf{7}}:$ les coefficients de régression relatifs aux soldes budgétaires. Chaque équation comporte les variables numérotées de 1 à 7 .

Pour savoir si l'indépendance de la banque centrale a un impact sur le solde budgétaire, on étudie les coefficients suivants pour chaque équation :

- Si $\gamma_{1}>\boldsymbol{0}$ et $\boldsymbol{\delta}_{1}>\mathbf{0}$, alors l'indépendance légale de la banque centrale (LIBC) encourage le déficit budgétaire, et l'indépendance réelle (TOR) aggrave cet impact.

- Si $\gamma_{1}>\boldsymbol{0}$ et $\boldsymbol{\delta}_{1}<\boldsymbol{0}$, alors l'indépendance légale de la banque centrale (LIBC) encourage le déficit budgétaire, mais l'indépendance réelle (TOR) atténue cet effet.

- Si $\boldsymbol{\gamma}_{\mathbf{1}}<\boldsymbol{0}$ et $\boldsymbol{\delta}_{\mathbf{1}}<\boldsymbol{0}$, alors l'indépendance légale de la banque centrale (LIBC) décourage le déficit budgétaire, et l'indépendance réelle (TOR) accentue cet impact.

- Si $\boldsymbol{\gamma}_{\mathbf{1}}<\boldsymbol{0}$ et $\boldsymbol{\delta}_{\mathbf{1}}>\mathbf{0}$, alors l'indépendance légale de la banque centrale (LIBC) décourage le niveau de déficit budgétaire, mais l'indépendance réelle (TOR) réduit cet effet.

\section{Présentation et justification des variables}

a) Variable expliquée ou endogène

Dans le modèle 1 et 2 la variable expliquée est le solde budgétaire (Ln_DEBU $i, t)$. Il évalue l'orientation de la politique budgétaire au cours d'un exercice financier. Il est égal à la différence entre les recettes budgétaires hors dons et les dépenses budgétaires hors dépenses en capital financées par

${ }^{27}$ Comme Sikken et De Haan (1998), D’Amato et al. (2009) et Mpofu (2012), nous parlerons plus de déficits budgétaires en lieu et place de solde budgétaire dans notre analyse car, les déficits sont caractéristiques des pays en de développement. 
l'extérieur. Le solde budgétaire peut être excédentaire (solde positif $>0$ ), déficitaire (solde négatif $<0$ ) ou équilibré $(=0)$. Dans la zone CEMAC, il fait partir des critères de convergence de premier rang. Pour Guérineau et al. (2015), ce critère est au cœur du dispositif de surveillance multilatérale. En effet, plus un gouvernement influence l'autorité monétaire pour monétiser les déficits, plus le degré d'indépendance de la banque centrale est faible.

Graphique 1 : Evolution du solde budgétaire de base en \% du PIB dans la CEMAC entre 1994 et 2016

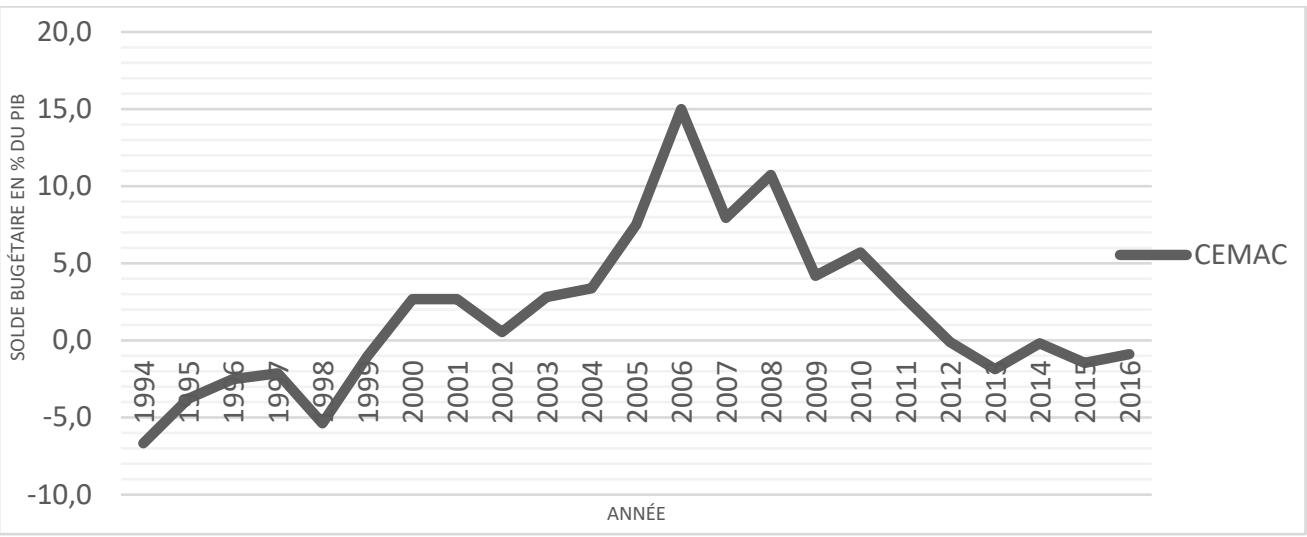

Source : Construction des auteurs à partir d'Excel 2016.

\section{Variables d'intérêts ou institutionnelles}

L'indice d'indépendance légale de la banque centrale $\left(\mathbf{L n} \_\mathbf{L I B C} \mathbf{i , t}\right)$. C'est le degré d'indépendance que le législateur confère à la banque centrale. Cukierman et al. (1992) précisent que le critère utilisé pour évaluer l'indépendance légale de la banque centrale dépend de la précision apportée aux textes des lois constitutionnelles concernant l'institut. Ainsi, les banques centrales sont considérées d'autant plus indépendantes sur le plan légal que la durée du mandat des dirigeants est longue, et que le pouvoir exécutif intervient légalement peu dans la nomination et le limogeage des dirigeants, ainsi que dans la conduite de la politique monétaire. De même, une banque centrale qui limite strictement ses avances au secteur public bénéficie de plus d'indépendance et peut interdire d'avoir recours à la " planche à billets " pour financer un déficit des comptes publics (elle incite de ce fait à une discipline budgétaire). Comme Bodea (2013) et Bodea et Higashijima (2017) nous prévoyons un signe négatif pour cette variable.

L'indice d'indépendance réelle de la banque centrale (Ln_TORi,t).

Il s'agit de la rotation des gouverneurs de banques centrales. Cet indice est obtenu en calculant le nombre de changement de gouverneurs au cours d'une période. En présupposant que le gouverneur aura du mal à mettre sur pied des politiques de long terme durant un mandat court, une rotation élevée des gouverneurs indique un faible niveau d'indépendance. Autrement dit, plus 
le mandat est long, moins les pressions politiques sont importantes ${ }^{28}$ et plus le taux de rotation est faible. Par conséquent, la banque centrale pourra renforcée son autonomie en impliquant une spécialisation de l'instrument monétaire dans la lutte contre l'inflation, et réduire la possibilité d'utilisation combinée des instruments budgétaire et monétaire (elle pourra ainsi affecter l'usage conjoncturel de l'instrument budgétaire). Comme Lucotte (2009), nous nous attendons à un signe négatif de la part de cette variable.

\section{Variables explicatives ou exogènes ou de contrôles}

Le taux d'ouverture commerciale (Ln_OUVi,t). Cette variable évalue l'intensité de l'intégration de l'économie nationale dans le commerce mondial et, dans une certaine mesure, la dépendance d'une économie sur la conjoncture internationale et sa sensibilité aux variations des prix internationaux. L'ouverture commerciale permet d'évaluer la force relative de la contrainte extérieure à laquelle l'économie est soumise et détermine en partie l'économie politique « espace de respiration », en particulier en matière de politique budgétaire (Blancheton, 2004). Par conséquent, un pays ayant un degré relativement élevé d'ouverture commerciale peut être plus vulnérable aux chocs extérieurs. Cette augmentation de la vulnérabilité n'est pas sans conséquence sur le solde budgétaire de l'Etat. Cependant, Combes et SaadiSedik (2006) mettent l'accent sur les résultats contradictoires concernant l'effet de l'ouverture commerciale sur les soldes budgétaires dans les études qui incluaient ce proxy de la vulnérabilité externe en tant que variable de contrôle pour les déficits budgétaires. En outre, bien que cette mesure de l'ouverture commerciale soit largement utilisée dans la littérature empirique, elle a été critiquée par certains économistes d'où l'incertitude relevé par rapport à son signe.

La masse monétaire (Ln_MAMO $\mathbf{i , t})$. Elle représente la monnaie et le quasi monnaie (M2) en pourcentage de PIB. Partant de l'hypothèse d'une politique monétaire accommodante, il existe une corrélation positive entre les déficits budgétaires élevés et croissance de la masse monétaire. Miller (1983) soutient que les déficits budgétaires peuvent générer une inflation unidirectionnelle par l'éviction de l'investissement privé en raison de taux d'intérêt élevés associés à ces déficits s'ils sont monétisés. On prévoit à cet effet un signe positif pour cette variable (Fry, 1998).

Le taux de croissance produit intérieur brut réel (Ln_TPIBi,t). Il représente la variation relative du produit intérieur brut réel par tête d'une année à l'autre. Le solde budgétaire d'un pays est sensible aux fluctuations économiques. En effet, lorsque le niveau de l'activité économique est faible

${ }^{28}$ A un niveau de rotation élevé donné, la durée du mandat du gouverneur est inférieure à celui du gouvernement, ce qui fragilise ce dernier, le décourageant à mener des politiques de long-terme. 
ou modéré, le montant des recettes fiscales collectées par le gouvernement diminue alors que les dépenses sociales augmentent d'où la détérioration de l'équilibre budgétaire. À l'inverse, une croissance économique plus élevée génère une amélioration du solde budgétaire (stabilisateur automatique). Ainsi, le signe de cette devrait être positif. Cependant, certains auteurs (voir par exemple Talvi et Végh, 2000) suggèrent que la politique budgétaire peut être pro cyclique dans les pays en développement avec des gouvernements laxistes ; car les pressions politiques pour accroître les dépenses publiques vont de pair avec les recettes fiscales croissantes en raison de la croissance économique. La forte augmentation des demandes fiscales au cours du boom économique s'appelle alors " l'effet de voracité » (Lane et Tornell, 1999). Ainsi, selon Woo (2003), le signe de ce coefficient est une question purement empirique.

Le taux d'inflation transformé (Ln_TINFLTRANS $i, t$ ). Il est défini comme le taux d'inflation divisé sur un plus le taux d'inflation pour le pays $\mathbf{i}$ à l'instant $\mathbf{t}$. Cette transformation ${ }^{29}$ est plus utile pour les études sur les marchés émergents et les pays en développement, qui sont caractérisées par des épisodes d'hyper inflation (Romelli, 2015). L'inflation est considérée par les économistes comme une contre-performance et par conséquent, augmente les déficits budgétaires. C'est pour cette raison que nous prévoyons un signe positif pour cette variable (Mpofu, 2012).

* La part d'agriculture dans le PIB (Ln_AGRi,t). Selon Tanzi (1992), la structure économique d'un pays est un facteur important qui pourrait influencer le niveau de taxation. C'est pour cette raison que la part de l'agriculture dans le PIB est incluse dans nos modèles. Néanmoins, le signe attendu de cette variable est incertain parce que la théorie distingue deux effets opposés de la part de l'agriculture dans le PIB sur la part des impôts. Ainsi, du côté de l'offre, on s'attend à ce que la part de l'agriculture dans le PIB ait un effet négatif sur les recettes fiscales, car les contraintes politiques pourraient encourager le gouvernement à réduire l'imposition dans ce secteur, souvent fortement taxé de manière implicite par des quotas d'importation, des tarifs, et des prix contrôlés pour la production ou des taux de change surévalués (Tanzi, 1992). En outre, le secteur agricole dans les pays en développement se caractérise principalement par l'agriculture de subsistance et la prédominance des petits agriculteurs. Il semble difficile pour un gouvernement d'imposer les principaux aliments utilisés pour la subsistance (Stotsky et Wolde, 1997). À l'inverse, du côté de la demande, on s'attend à ce que la part de l'agriculture

${ }^{29}$ L'utilisation de l'inflation transformée permet de réduire l'hétéroscédacticité des erreurs et d'augmenter l'efficacité des estimateurs car, en utilisant l'inflation régulière on pourrait avoir un poids inflationnistes excessifs et des valeurs aberrantes dans notre échantillon (Cukierman et al. (1992), De Haan et Kooi (2000), Neyapti (2003), Jacome et Vazquez (2008), Arnone et al. (2008)). 
dans le PIB ait un effet positif sur l'excédent budgétaire car, de nombreuses activités du secteur public sont axées sur la ville (la demande de biens et de services publics) de sorte que les dépenses publiques sont théoriquement réduites (Teera, 2003).

Le taux d'urbanisation $\left(\right.$ Ln_TXURB $\left._{\mathbf{i}, \mathbf{t}}\right)$. Cette variable est introduite dans les modèles car, Edwards et Tabellini (1991) montrent qu'il est relativement plus facile et moins coûteux pour les autorités fiscales de collecter des taxes dans les zones urbaines avec une concentration plus élevée du secteur formel que dans des zones rurales, où le degré d'évasion fiscale est particulièrement élevé (Ansari, 1982). On s'attend alors à ce que cette variable ait un signe négatif (diminution du déficit budgétaire).

\section{Méthode d'estimation : la technique des données de panel}

L'estimation des deux modèles se fera à travers la technique des données de panel non dynamique (panels statiques) utilisée récemment par Tangakou et al. (2015) et Noula et al. (2016). Les données de panel offrent un avantage incontournable parce qu'elles prennent en compte au moins deux dimensions : individuelle et temporelle. Elles contiennent des données sur plusieurs individus observés sur plusieurs dates. L'utilité des données de panel est qu'elles permettent de contrôler trois types de facteurs : Ceux qui varient entre les individus, mais ne varient pas au cours du temps ; ceux qui pourraient causer un biais d'omission si l'on n'en tenait pas compte ; ceux qui sont inobservables ou non disponibles et ne peuvent être inclus dans la régression.

Mais avant tout, quelques tests préliminaires s'avèrent nécessaires à l'application de l'économétrie de données de panel (Tests de stationnarité, test d'omission de Ramsey-Reset, test Variance Inflation Factor (VIF), test d'homogénéité, test de Hausman, test d'hétéroscédasticté de Breush-Pagan, test d'autocorrélation de Wooldridge, test d'endogénéité).

\section{Présentation des résultats et enseignements Résultats des tests}

Dans le cadre de la stationnarité, nous allons émettre deux (02) hypothèses à savoir :

H0 : Absence de stationnarité ; H1 : Présence de stationnarité.

La synthèse des résultats des tests de stationnarité est représentée dans le tableau de l'annexe 2. De ces tests, il en ressort que toutes les séries retenues sont stationnaires. Nous passons à présent à la synthèse des tests préliminaires et aux résultats de l'estimation de nos équations budgétaires par la méthode des Moindres Carrés Généralisés Faisables (MCGF) (voir les tableaux 2 et 3 ci-dessous). 
Tableau 2 : Présentation de la synthèse des tests économétriques préliminaires

\begin{tabular}{|c|c|c|c|c|c|}
\hline Types de Test & Hypothèse nulle & \multicolumn{2}{|c|}{ P-value } & Acceptation & Conséquence \\
\hline Modèle 1 et Modèle 2 & Modèle 1 et 2 & $\begin{array}{c}\text { Modèle } \\
1\end{array}$ & $\begin{array}{c}\text { Modèle } \\
2\end{array}$ & $\begin{array}{l}\text { Modèle } 1 \text { et } \\
2\end{array}$ & Modèle 1 et 2 \\
\hline $\begin{array}{l}\text { 1-Test d'omission de } \\
\text { Ramsey-Reset }\end{array}$ & $\begin{array}{l}\text { Le modèle n'a pas } \\
\text { omis des variables } \\
\text { explicatives } \\
\text { pertinentes }\end{array}$ & 0.2878 & 0.2878 & Accepter & $\begin{array}{l}\text { Pas de variables } \\
\text { pertinentes omis }\end{array}$ \\
\hline $\begin{array}{c}\text { 2- Test Variance } \\
\text { Inflation Factor (VIF) }\end{array}$ & $\begin{array}{l}1 \text { / VIF doit être } \\
\text { supérieur à } 0.1\end{array}$ & 0.4490 & 0.4490 & Accepter & $\begin{array}{c}\text { Absence des } \\
\text { problèmes de } \\
\text { multi-colinéarité }\end{array}$ \\
\hline $\begin{array}{l}\text { 3- Test d'homogénéité } \\
\text { globale }\end{array}$ & $\begin{array}{l}\text { Pas d'effets } \\
\text { spécifiques }\end{array}$ & 0.0586 & 0.0586 & Accepter & $\begin{array}{c}\text { Les effets } \\
\text { individuels ne sont } \\
\text { pas nécessaires }\end{array}$ \\
\hline 4- Modèle à effets fixes & $\begin{array}{l}\text { Pas de différence } \\
\text { systématique entre } \\
\text { les coefficients }\end{array}$ & 0.1059 & 0.1059 & Rejet & $\begin{array}{c}\text { Modèle à effet fixe } \\
\text { non significatif }\end{array}$ \\
\hline $\begin{array}{l}\text { 5- Modèle à effets } \\
\text { aléatoires }\end{array}$ & $\begin{array}{l}\text { Pas de différence } \\
\text { systématique entre } \\
\text { les coefficients }\end{array}$ & 0.0017 & 0.0017 & Accepter & $\begin{array}{l}\text { Le modèle à effet } \\
\text { aléatoire est } \\
\text { significatif à } 1 \%\end{array}$ \\
\hline 6- Test de Hausman & $\begin{array}{c}\text { Absence de } \\
\text { corrélation entre les } \\
\text { erreurs et les } \\
\text { variables } \\
\text { explicatives }\end{array}$ & 0.1146 & 0.1440 & Accepter & $\begin{array}{l}\text { Le modèle à effets } \\
\text { aléatoires est } \\
\text { approprié }\end{array}$ \\
\hline $\begin{array}{c}\text { 7- Test } \\
\text { d'hétéroscédasticité de } \\
\text { Breush-Pagan }\end{array}$ & $\begin{array}{c}\text { Absence } \\
\text { d'hétéroscédasticité }\end{array}$ & 0.0030 & 0.0030 & Rejet & $\begin{array}{c}\text { Utilisation des } \\
\text { Moindres Carré } \\
\text { Généralisés (MCG) }\end{array}$ \\
\hline $\begin{array}{c}\text { 8- Test d'auto- } \\
\text { corrélation intra- } \\
\text { individus de Wooldridge }\end{array}$ & $\begin{array}{l}\text { Absence d'auto- } \\
\text { corrélation } \\
\text { des erreurs }\end{array}$ & 0.1668 & 0.1668 & Accepter & $\begin{array}{l}\text { La structure n'est } \\
\text { pas auto-corrélée }\end{array}$ \\
\hline 9- Test d'endogénéité & $\begin{array}{c}\text { Absence } \\
\text { d'endogénéité }\end{array}$ & 0.276 & 0.276 & Accepter & $\begin{array}{l}\text { Exogénéité des } \\
\text { variables } \\
\text { suspectées }\end{array}$ \\
\hline
\end{tabular}

Source : Construction des auteurs à partir de Stata 13.

Tableau 3 : Résultats des effets de l'indépendance légale (modèle 1) et réelle (modèle 2) de la BEAC sur la discipline budgétaire dans la CEMAC par la méthode des MCGF

\begin{tabular}{|c|c|c|c|c|c|c|c|}
\hline \multirow[b]{2}{*}{$\begin{array}{l}\text { Modèle } 1 \\
\text { Modèle } 2\end{array}$} & \multicolumn{7}{|c|}{ Variables expliquées ou endogènes } \\
\hline & $\begin{array}{c}\text { Variables } \\
\text { explicatives } \\
\text { ou } \\
\text { exogènes }\end{array}$ & coefficients & $\begin{array}{l}\text { Standard } \\
\text { error }\end{array}$ & $\mathrm{z}$ & $\begin{array}{l}\mathrm{P}> \\
|\mathrm{z}|\end{array}$ & & $\begin{array}{l}\text { [95\% Conf. } \\
\text { Interval] }\end{array}$ \\
\hline Modèle 1 & $\log \_$libc & -50.63767 & 90.6353 & -0.56 & $0.576-2$ & 228.279 & 127.004 \\
\hline Modèle 2 & log_tor & 56.89637 & 101.8376 & 0.56 & $0.576-1$ & 142.701 & 256.494 \\
\hline Modèle 1 & log_ouv & 0.005302 & 0.01148 & 0.46 & $0.644 \quad-0$ & 0.01721 & 0.02781 \\
\hline Modèle 2 & log_ouv & 0.005302 & 0.01148 & 0.46 & $0.644 \quad-0$ & 0.01721 & 0.02781 \\
\hline Modèle 1 & $\log \_$mamo $^{a}$ & a -0.581046 & 0.19748 & -2.94 & 0.003 & -0.9681 & -0.19398 \\
\hline Modèle 2 & $\log _{\text {mamo }}{ }^{a}$ & a $\quad-0.581046$ & 0.19748 & -2.94 & 0.003 & -0.9681 & -0.19398 \\
\hline Modèle 1 & $\log$ tpib & -0.00321 & 0.04081 & -0.08 & $0.937-0$ & .08322 & 0.07678 \\
\hline
\end{tabular}




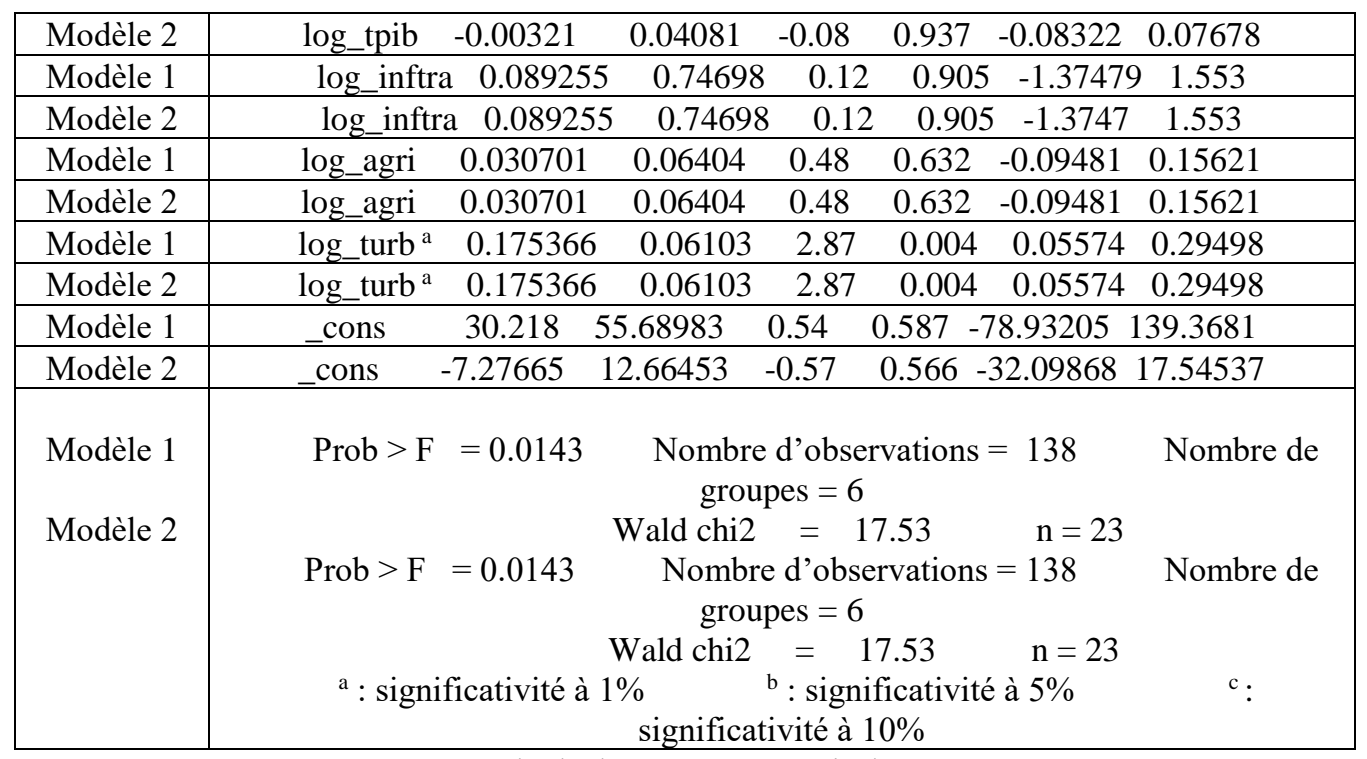

Source : Calculs des auteurs à partir de Stata 13.

\section{Interprétations économiques}

Nos modèles 1 et 2 sont de bonnes qualités en générale. Le tableau 3 nous informe qu'ils sont globalement significatifs au seuil de 5\% car (Prob > $\mathbf{F}=\mathbf{0 . 0 1 4 3}$ ) est inférieur à $5 \%$. Partant de là, plusieurs interprétations sont faites.

Les résultats des estimations indiquent que le degré d'indépendance légale $\left(\log _{-} \mathbf{L I B C} \mathbf{i}, \mathbf{t}\right)$ de la BEAC réduit les déficits budgétaires dans la zone CEMAC car, le signe de son coefficient est négatif (-50.63767) et conforme à nos prédictions. Mais malheureusement, cette variable est non significative (modèle 1). Cela nous fait dire qu'une augmentation d'une unité du degré d'indépendance légale de la BEAC, entraine une diminution du déficit budgétaire de 50.63767 fois cette unité. Il existerait alors une relation négative et non significative entre ces deux variables. Ce résultat est en harmonie avec ceux de Bénassy-Quéré et Pisani-Ferry (1994), Sikken et De Haan (1998) et D'Amato et al. (2009). Il peut s'interpréter comme une absence de contrainte sur la politique budgétaire, ou comme la prise en charge partielle de la stabilisation conjoncturelle par la BEAC même lorsque celle-ci est indépendante. Il peut aussi s'interpréter comme une absence d'obligation pour la politique budgétaire de prendre en charge le réglage conjoncturel lorsque la Banque Centrale vise la stabilité des prix. Enfin, ce résultat mentionne également le manque de rigueur dans le respect des textes (statuts de la BEAC) que l'on observe généralement dans les pays en développement (Cukierman et al., 1992). 
Le coefficient du taux de rotation des gouverneurs de la BEAC $\left(\log _{-}\right.$TOR $\left.\mathbf{R}_{\mathrm{i}, \mathrm{t}}\right)\left(\mathbf{+ 5 6 . 8 9 6 3 7 ^ { 3 0 }}\right)$ est positif et contraire à nos attentes (modèle 2). Cette variable est également non significative. Par ce résultat, une unité supplémentaire du degré d'indépendance réelle de la Banque Centrale va entrainer une augmentation du déficit budgétaire de $\mathbf{5 6 . 8 9 6 3 7}$ fois cette unité d'où, l'existence d'un lien positif entre ces deux variables. Ce résultat en contradiction avec nos attentes, rejoint celui de Mpofu (2012). En effet, certains aspects informels et comportementaux de la relation entre la BEAC et les gouvernements de la CEMAC peuvent justifier ce résultat (les conventions entre la Banque Centrale et les Etats, les influences politiques, la qualité du Conseil d'administration de la Banque Centrale, la personnalité des individus à la tête de la Banque Centrale et la soumission ou non des Autorités Monétaires aux gouvernements).

Le coefficient de la variable ouverture commerciale $\left(\log _{-} \mathbf{O U} \mathbf{V}_{\mathbf{i}, \mathbf{t}}\right)$ est positif (+0.005302) mais, reste non significatif (modèle $\mathbf{1}$ et $\mathbf{2}$ ). La hausse d'une unité de l'ouverture commerciale va entrainer une augmentation du déficit budgétaire de $\mathbf{0 . 0 0 5 3 0 2}$ fois cette unité. A l'instar de Lucotte (2009), il apparait alors une relation positive entre ces deux variables. Ce résultat mentionne la vulnérabilité des pays de la CEMAC aux chocs externes tels que la chute marquée des cours du pétrole qui perturbe profondément les soldes extérieurs et budgétaires de la Région.

La variable masse monétaire $\left(\log _{-} \mathbf{M A M O}_{\mathrm{i}, \mathrm{t}}\right)$ est significative à $1 \%$ (modèle 1 et 2). Le signe négatif de son coefficient (-0.581046) est inattendu. L'augmentation d'une unité de la masse monétaire va conduire à une baisse du déficit budgétaire de $\mathbf{0 . 5 8 1 0 4 6}$ fois cette unité d'où, une relation inverse entre ces variables. Comme Brown et Yousefi (1996), nous ne trouvons pas de preuves que les déficits monétaires sont monétisés. Ce résultat peut provenir des stratégies anti-inflationnistes mis en place par le BEAC depuis 1994.

Le PIB (Log_TPIB $\mathbf{i , t})$ a un coefficient négatif (-0.00321) dans nos deux modèles. Il apparait dans ce contexte un impact négatif du PIB sur le déficit budgétaire mais, cette action est non significative. Un surplus d'une unité du PIB entrainera une baisse du déficit budgétaire de $\mathbf{0 . 0 0 3 2 1}$ fois cette unité. Comme le souligne Talvi et Végh (2000), la politique budgétaire peut être pro cyclique dans les pays en développement et particulièrement dans la CEMAC où, la surveillance multilatérale a contribué à renforcer le biais pro cyclique de la politique budgétaire. Toutefois, un autre facteur peut justifier ce comportement pro cyclique notamment, la forte augmentation des recettes

${ }^{30}$ La valeur élevée des coefficients des variables LIBC et TOR s'explique par une faible variation de ces derniers sur la période de l'étude. 
pétrolières durant la dernière décennie qui a amené les Etats à augmenter significativement leurs dépenses (Bikai, 2015).

Le coefficient de l'inflation transformée $\left(\log _{\mathbf{I N F T R}} \mathbf{I}, \mathbf{t}\right)$ est positif (+0.089255) conformément à nos prédictions, mais il reste non significatif (modèle 1 et 2). L'augmentation d'une unité de l'inflation transformée va entrainer une hausse du déficit budgétaire de $\mathbf{0 . 0 8 9 2 5 5}$ fois cette unité. Il existerait dès lors une relation positive entre ces deux variables. Ce résultat rejoint celui de Neyapti (2003) qui précise que généralement le gouvernement crée l'inflation car, il a recours tôt ou tard à la création monétaire pour financer les déficits budgétaires croissants.

La part d'agriculture dans le PIB (Log_AGRI $\mathbf{I}_{\mathbf{i}, \mathbf{t}}$ ) est non significative

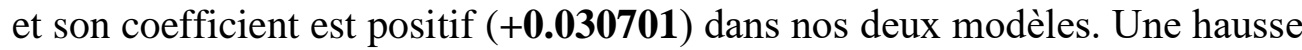
d'une unité de la part de l'agriculture dans le PIB déclenchera une augmentation du déficit budgétaire de $\mathbf{0 . 0 3 0 7 0 1}$ fois cette unité. La part d'agriculture dans le PIB encouragerait ainsi les déficits dans la CEMAC. En effet, comme l'ont souligné Stotsky et Wolde (1997), il semble difficile pour les gouvernements des pays en développement qui se caractérise principalement par une agriculture embryonnaire et la prédominance des petits agriculteurs d'imposer une taxe aux principaux aliments utilisés pour la subsistance des populations.

Le coefficient du taux d'urbanisation $\left(\log _{-}\right.$TURB $\left.\mathbf{i}, \mathbf{t}\right)$ est positif (+0.1753665) et significatif à $1 \%$ (modèle 1 et 2 ). Le taux d'urbanisation accentue le déficit budgétaire dans la CEMAC. Une augmentation d'une unité du taux d'urbanisation va entrainer une hausse du déficit budgétaire de $\mathbf{0 . 1 7 5 3 6 6 5}$ fois cette unité. Ce résultat peut s'expliquer par un degré d'évasion fiscale particulièrement élevé pas seulement en zone rural comme le constate Ansari (1982), mais aussi dans les zones urbaines, où la corruption et les détournements de fonds publics sont les plus réguliers dans les pays de voie de développement.

Le tableau 4 ci-dessous fait un récapitulatif entre les signes attendus et les résultats obtenus dans notre analyse.

Tableau 4 : Tableau de comparaison entre les signes attendus et les signes obtenus.

\begin{tabular}{|c|c|c|}
\hline Variables & Signes attendus & Signes obtenus \\
\hline $\log \_L I B C_{i, t}$ & - (négatif) & - (négatif) \\
\hline${\log \_\mathrm{TOR}_{\mathrm{i}, \mathrm{t}}}$ & - (négatif) & $+\quad$ (positif) \\
\hline $\log \_O U V_{i, t}$ & $+/$ - (incertain) & $+\quad$ (positif) \\
\hline $\log _{2} M_{A M O}, t+\mathrm{t} * *$ & + (positif) & - (négatif) \\
\hline $\log _{2}$ TPIB $_{\mathrm{i}, \mathrm{t}}$ & $+/$ - (incertain) & - (négatif) \\
\hline $\log \_I N F T R A_{i, t}$ & $+\quad$ (positif) & $+\quad$ (positif) \\
\hline $\log _{-} A G R I_{i, t}$ & +/- (incertain) & + (positif) \\
\hline $\log _{-}$TURB $_{\mathrm{i}, \mathrm{t}} * * *$ & - (négatif) & $+\quad$ (positif) \\
\hline Rapport de validation & \multicolumn{2}{|c|}{ Soit $25 \%$} \\
\hline Décision & \multicolumn{2}{|c|}{ Faible significativité } \\
\hline
\end{tabular}

Source : Construction des auteurs. 


\section{Conclusion}

Depuis la crise économique de 2007, 1'indépendance de la banque centrale est de nouveau un sujet très débattu. A cet effet, nombreux arguments peuvent être avancés pour justifier le lien entre le degré d'indépendance de la banque centrale et la discipline budgétaire, dans le cas particulier des économies les moins avancées. Malgré cela, très peu d'études empiriques se sont attachées jusqu'à aujourd'hui à tester la validité de ce lien dans ce contexte. La recherche conduite dans cet essai visait précisément à combler ce vide dans la littérature en évaluant l'impact de l'indépendance de la Banque des Etats de 1'Afrique Centrale (BEAC) sur les déficits budgétaires dans la zone CEMAC entre 1994 et 2016.

Après avoir montré que la BEAC est une banque centrale indépendante en mesurant son degré d'autonomie légale et réelle entre 2010 et 2016, et 1994 et 2016 respectivement, deux modèles économétriques inspirés des travaux théoriques de Barro (1989) relatif au solde budgétaire ont été spécifiés. L'estimation à l'aide du logiciel Stata 13 de nos modèles empiriques nous a conduits à adopter la méthode des Moindres Carrés Généralisés Faisables appliquée aux données de panels statiques.

Les résultats que nous avons obtenus à l'issue de nos estimations mettent en lumière un impact mitigé et non significatif de l'indépendance légale et réelle de la BEAC sur la discipline budgétaire dans cette Zone. Ces résultats nous confortent puisqu'ils s'inscrivent dans la lignée de certains travaux empiriques ayant étudié ce lien dans les économies émergentes, et confirment néanmoins que l'orientation moyenne de la politique budgétaire est davantage déterminée par la procédure et la manœuvre budgétaire. Plusieurs travaux ont montré que les différences de comportement budgétaire entre les Etats sont avant tout fonction de leur structure (unitaire ou fédérale), des règles qu'ils se sont fixées pour la préparation du budget, des procédures de discussion et de vote du budget par le Parlement, et de leur degré de discipline dans l'exécution des lois de finances, ainsi que des conditions sociopolitiques générales du pays. Ces facteurs sont nettement plus importants que l'effet de 1'autonomie de la banque centrale. Toutefois, la fragilité de ces résultats est sous-entendue. Pour être complet, il aurait en outre fallu tenir compte d'autres contraintes de type monétaire comme le régime de change ou encore le déflateur du PIB.

\section{References:}

1. Aisen A. et Veiga F J. (2008). The political economy of seigniorage. Journal of Development Economics ; $\mathrm{n}^{\circ}$ 87(1), pp29-50.

2. Alesina A. et Summers L. (1993). Central bank independence and macroeconomic performance : Some comparative evidence. Journal of Money, Credit and Banking, pp.151-162. 
3. Alesina A. et Tabellini G. (1987). Rules and discretion with noncoordinated monetary and fiscal policies. Economic Inquiry 25(4) ; pp.619-630.

4. Ansari M M. (1982). Determinants of tax ratio : a cross-country analysis. Economic and Political Weekly, June 19, pp.1035-1042.

5. Arnone M., Laurens J., Segalotto J. et M. Sommer M. (2008). Central bank autonomy : lessons from Global Trends. IMF Working Paper, No. $07 / 88$.

6. Avom D. et Bobbo A. (2013). La BEAC en quête de son autonomie. Revue d'économie financière, Juin, pp. 125-144.

7. Banaian K R. (2008). Measuring central bank independence : ordering, ranking, or scoring ? dans Banain, K. et Roberts, B. (Eds). The design and use of political economy indicators. Palgrave Mac millan.

8. Barro R J. (1974). Are government bonds net wealth ? Journal of Political Economy ; n82 (6), pp.1095-1118.

9. Batini N. et Laxton D. (2006). Under what conditions can inflation targeting be adopted ? the experience of emerging markets. Working paper Central Bank of Chile 406, Central Bank of Chile.

10. Beetsma R. et Bovenberg L. (1997). Central bank independence and public debt policy. Journal of Economic Dynamics and Control $\mathrm{n}^{\circ}$ 21(4-5), 873-894.

11. Beetsma R. et Bovenberg L. (1998). Monetary union without fiscal coordination may discipline policymakers. Journal of International Economics ; ${ }^{\circ} 45(2)$, pp.239-258.

12. Beetsma R. et Bovenberg L. (1999). Does monetary unification lead to excessive debt accumulation ? Journal of Public Economics $\mathrm{n}^{\circ}$ 74(3), pp.299-325.

13. Bénassy-Quéré A. et Pisani-Ferry J. (1994). Indépendance de la banque centrale et politique budgétaire. CEPII, document de travail $\mathrm{n}^{\circ}$ 94-02.

14. Bensafta K M. (2011). Eléments de la défiance britannique vis-à-vis de l'Euro et de l'EUM : rôle des facteurs monétaire. Thèse de doctorat en sciences économiques. Université François-Rabelais de Tours. 27 Juin 2011.

15. Berger H., De Haan J. et Eijffinger S. (2001). Central bank independence : an update of theory and evidence. Journal of Economic Surveys, $\mathrm{n}^{\circ} 15$, pp. 3-40.

16. Bergin (2000). Fiscal solvency and price level determination in a monetary union. Journal of Monetary Economics, 2000, vol. 45, issue $1 ;$ pp.37-53. 
17. Bessma Monani et Samantha St. Armand (2014). Central bank independence in North Africa. The Center for International Governance Innovation- CIGI (Mars 2014).

18. Bikai L. (2015). Règle de surveillance multilatérale et pro cyclicité de la politique budgétaire dans la zone CEMAC. BEAC Working Paper, BWP N 03/15.

19. Blancheton B. (2004). Ouverture commerciale, croissance et développement : malentendus et ambiguïtés des débats. Journée du développement du GRES, University Montesquieu-Bordeaux IV, September.

20. Bodea C. (2013). Independent central banks, regime type and fiscal performance : the case of Post-communist countries. Public Choice ; $\mathrm{n}^{\circ} 155(1-2)$; pp.81-107.

21. Bodea C. et Higashijima M. (2017). Central Bank Independence and Fiscal Policy : incentives to spend and constraints on the executive. British Journal of Political Science ; $\mathrm{n}^{\circ} 47(1)$, pp.47-70.

22. Burdekin R. et Laney L. (1988). Fiscal policy making and the central bank institutional constraint. Kyklos ; ${ }^{\circ} 41(4)$, pp.647-662.

23. Buderkin R. et Laney L. (2015). Fiscal policy making and the central Bank Institutional contraint una vez mas : new Latin American evidence. Hawaï pacific University-Juillet 2015.

24. Calvo G. (1989). Controlling inflation : the problem of non-indexed debt, dans Edwards S. et Larrain, F. (Eds.) Debat, Adjustment and Recovery : Latin America's prospect for growth and development. Blackwell, New York, pp.156-175.

25. Catão L. et Terrones M. (2005). Fiscal deficits and inflation. Journal of Monetary Economics n ${ }^{\circ}$ 52(3), pp.529-554.

26. Christiano J. et Fitzgerald J. (2000). Understanding the fiscal theory of the price level. Federal Reserve Bank of Cleveland Economic Review ; n³6(2), pp.2-38.

27. Combes J-L. et Saadi-Sedik T. (2006). How does the trade openness influence budget deficits in developing countries. Journal of Development Studies $\mathrm{n}^{\circ} 46$ (8), pp.1401-1416.

28. Crowe C. et Meade E. (2008). Central bank independence and transparency : evolution and effectiveness. European Journal of Political Economy, n²4 (4), pp.763-777.

29. Cukierman A., Webb S., et Neyapti B. (1992). Measuring the independence of central banks and its effects on policy outcomes. The World Bank Economic Review, 6(3), pp. 353-398.

30. Cushing J. (1999). The indeterminacy of prices under interest rate pegging : the nonricardian case. Journal of Monetary Economics ; n $44(1)$, pp.131-148 D.C. 
31. D'Amato B., Pistoresi D. et Salsano F. (2009). The determinants of central bank independence. Journal of Finance and Economics, $\mathrm{n}^{\circ} 14$, pp. 107-119.

32. Davig T., Leeper M. et Walker B. (2011). Inflation and the fiscal limit. European Economic Review ; ${ }^{\circ} 55(1), \mathrm{pp} 31-47$.

33. De Haan J. et J. E. Sturm (1992). The case for central bank independence. Banca Nazionale del Lavoro Quarterly Review; $\mathrm{n}^{\circ} 182$, pp. 305-327.

34. De Haan J. et Kooi W. (2000). Does central bank independence really matter ? new evidence for developing countries using a new indicator. Journal of Banking and Finance, n²4, pp.643-664.

35. Debelle G. et Fischer S. (1994). How independent should a central bank be ? In J. Fuhrer (ed.), goals, guidelines and constraints facing monetary policymakers, Federal Reserve Bank of Boston, Conference vol.38; pp.195-221.

36. Edwards S. et Tabellini G. (1991). Explaining fiscal policies and inflation in developing countries. Journal of International Money and Finance ; ${ }^{\circ} 10$ (s1), pp.16-48.

37. Ehrhart H., Minea A. et Villieu P. (2009). Deficit, seigniorage and the growth Laffer curve in developing countries. Etudes et Documents 2009. $\mathrm{n}^{\circ} 26$, CERDI.

38. Eijffinger W. et De Haan J. (1996). The political economy of central bank independence. Special Papers in International Economics, No.19, International Finance Section, Department of Economics, Princeton University.

39. Eijffinger W. et Schaling E. (1992). Central bank independence : criteria and indices. Research Memorandum 548, Tilburg University.

40. Franta M., Libich J. et Stehlik P. (2011). The big picture of monetary-fiscal interactions. Economic Papers ; n $30(1)$, pp6-14.

41. Franzese R. (1999). Partially independent central banks, politically responsive governments, and inflation. American Journal of Political Science n ${ }^{\circ} 43$; pp.681-706.

42. Franzese R. (1999). Two hands on the wheel : independent central banks, politically responsive governments and inflation. www.personal.umich.edu/franzese.

43. Franzese R. (2002). Macroeconomic policies of developed democracies. Cambridge University Press.

44. Fry M. (1998). Assessing central bank independence in developing countries : do actions speak louder than words. Oxford Economic Papers, $\mathrm{n}^{\circ}$ 50, pp.512-529. 
45. Garriga A C. (2016). Central bank independence in the world : a new data set. International interactions. May 2016; Centro de Investigacion y Docencien economicas (CIDE).

46. Garriga A C. et Rodriguez C M. (2017). Stepping up during elections : independance centrals and inflation. 75th Midwest Political Science Association Annual Convention; April 6-7, Chicago I.L (CIDE).

47. Grilli D., Masciandaro G., et Tabellini E. (1991). Political and monetary institutions and public financial policies in the industrial countries. Economic Policy Review, n6(2), pp. 341-392.

48. Guérineau S., Guillaumont J S. et Léon F. (2015). Viabilité et renforcement du dispositive de surveillance multilatéral au sein de la CEMAC. Rapport FERDI, Juillet 2015.

49. Huang H. et Wei S-J. (2006). Monetary policies for developing countries : the role of institutional quality. Journal of International Economics ; n70(1), pp.239-252.

50. Jacome L. et Vazquez F. (2008). Is there any link between legal central bank independence and inflation ? evidence from Latin America and the Caribbean. European Journal of Political Economy; $\mathrm{n}^{\circ} 24$ (4), pp.788-801.

51. Karass A. (1994). Macroeconomic effects of budget deficits : further international evidence. Journal of International Money and Finance, $\mathrm{n}^{\circ} 13$, pp. 190-210.

52. Keefer P. et Stasavage D. (2003). The limits of delegation : veto players, central bank independence and the credibility of monetary policy, American Political Science Review ; n97, pp.407-423.

53. Kydland F. et Prescott E. (1977). Rules rather than discretion : the inconsistency of optimal plans. Journal of Political Economy, 85, pp.473-491.

54. Lane P. et A. Tornell A. (1999). Voracity Effect. American Economic Review n॰89 (1), pp.22-46.

55. Leeper M. et Walker B. (2011). Fiscal limits in advanced economies. Economic Papers; n³0(1), pp.33-47.

56. Lucotte Y. (2009). The influence of central bank independence on budget deficits in developing countries : new evidence from panel data analysis. Université d'Orléans, UMR CNRS 6221.

57. Lucotte Y. (2012). Etudes des interactions entre les stratégies de ciblage d'inflation et leur contexte institutionnel : application aux économies émergentes. Thèse de Doctorat en Science Economique soutenue le : 11 Décembre 2012. Université d'Orléans. 
58. Mhamdi Ghrissi (2009). Evaluation du degré d'indépendance de la banque centrale de Tunisie. Conference Paper April 2009 www.researchgate.net/publication/262301266.

59. Miller C. (1993). Fiscal deficits and interest rates : comment. Australian Economic Papers.

60. Minea, A., Tapsoba, R. et Villieu, P. (2012). Can inflation targeting promote institutional quality in developing countries?, mimeo.

61. Mpofu S. (2012). Essays on central bank independence and macroeconomic performance : selected african economies. The Faculty of Commerce, Law and Management, University of the Witwatersrand, Johannesburg, in Fulfillment of the Requirements for the Degree of Doctor of Philosophy, April 2012.

62. Neyapti B. (2003). Budget deficits and inflation : the roles of central bank independence and financial market development. Contemporary Economic Policy, n²1, October 2003, pp.458-475.

63. Ngniado N E. (2016). Incertitude et mise en œuvre de la politique monétaire dans la CEMAC. Economies et finances. Thèse de doctorat Université de Bordeaux, 2016.

64. Noula A G., Bkwayep R. et Mba F. (2016). Influence de la bancarisation et du crédit bancaire sur la croissance économique en zone CEMAC. European scientific journal. November (2016), Edition vol. $12 \mathrm{n}^{\circ} 11$.

65. Pollard P. (1993). Central bank independence and economic performance. Federal Reserve Bank of St. Louis. pp. 21-36.

66. Rogoff K. (1985). Can international monetary policy cooperation be counter productive. Journal of International Economics, 18, pp.199-217.

67. Romelli D. (2015). Three essays on central banking. Thèse Doctorat en Economie et Finances ; Université de Cergy pontoise, Décembre 2015.

68. Sadeh Tal. (2011). Hard currencies for hards times : terror attacks of monetary anchors. Defence and Peace Economics.

69. Sargent T. et Wallace N. (1981). Some unpleasant monetarist arithmetic. Federal Reserve Bank Minneapolis Quarterly Review (automne), pp.1-17.

70. Sargent T. (1985). Reaganomics and credibility, dans Ando. A., Eguchi, H., Farmer, R. et Suzuki, Y. (Eds.), Monetary Policy in our Times. MIT Press, Cambridge, pp.235-252.

71. Sikken B. et De Haan J. (1998). Budget deficits, monetization and central bank independence in developing. Oxford Economic Papers ; n50 (3), pp.493-511. 
72. Sims C.(1994). A simple model for study of the determination of the price level and the interaction of monetary and fiscal policy. Economic Theory $\mathrm{n}^{\circ} 4(3)$, pp.381-399.

73. Stotsky G. et Wolde Mariam A. (1997). Tax effort in sub-saharan africa. IMF Working Paper, WP/97/107.

74. Tabellini G. (1986). Central bank reputation and the monetization of deficits : the 1981 Italian Monetary Reform. Economic Inquiry, pp.185-200.

75. Tabellini G. (1986). Money, debt and deficits in a dynamic game. Journal of Economic Dynamics and Control ; ${ }^{\circ} 10(4), \mathrm{pp} .427-442$.

76. Talvi E. et Végh C A. (2000). Tax base variability and procyclical fiscal policy. NBER Working Paper No. 7499, National Bureau of Economic Research, Cambridge.

77. Tangakou S., Mba Fokwa et Merci B. (2015). Gouvernance bancaire et rentabilité financière au Cameroun. European scientific journal. Janvier 2015 édition; vol.11 $\mathrm{N}^{\circ} 1$.

78. Tanzi V. (1992). Structural factors and tax revenue in developing countries : a decade of evidence, in Open Economies : Structural Adjustment and Agriculture, Ed. by Goldin, I. and A.L. Winters, Cambridge University Press, Cambridge, pp.267-281.

79. Teera M. (2003). Determinants of taxe revenue share in uganda. Centre for Public Economics-Working Paper 09-03, University of Bath.

80. Van Aarle B., Bovenberg L. et Raith M. (1995). Monetary and fiscal policy interaction and government debt stabilization. Journal of Economics ; n62(2), pp.111-140.

81. Villieu P. (2011). Quel objectif pour la dette publique à moyen terme ? Revue d'Economie Financière ${ }^{\circ} 103(3)$, pp.79-98.

82. Woo J. (2003). Economic, political, and institutional determinants of public deficits. Journal of Public Economics ; n ${ }^{\circ} 87$, pp.387-426.

83. Woodford M. (1996). Control of the public debt : a requirement for price stability ? NBER Working Paper 5684; National Bureau of Economic Research, Cambridge. 


\section{ANNEXES}

Annexe 1 : Taux de rotation (TOR) des gouverneurs de la BEAC entre 1994 et 2016

\begin{tabular}{|c|c|}
\hline \multicolumn{2}{|c|}{ Période allant de 1994 à 2016} \\
\hline Gouverneur & $\begin{array}{c}\text { Taux de rotation du gouverneur = nombre de } \\
\text { changement par durée du mandat }\end{array}$ \\
\hline $\begin{array}{c}\text { J.F Mamalepot } \\
\text { (de } 1994 \text { au } 1^{\text {er }} \text { Août 1995) }\end{array}$ & $1 / 2=0.5$ \\
\hline $\begin{array}{c}\text { J.F Mamalepot } \\
\left(\text { du } 1^{\text {ier }} \text { Août } 1995 \text { au } 1^{\mathrm{er}}\right. \\
\text { Août 2000) }\end{array}$ & $1 / 5=0.2$ \\
\hline $\begin{array}{c}\text { J.F Mamalepot } \\
\text { (du } 1^{\text {ier }} \text { Août } 2000 \text { au } 20 \text { Juin } \\
\text { 2007) } \\
\end{array}$ & $1 / 7=0.14$ \\
\hline $\begin{array}{c}\text { P. Andzembé } \\
\text { (du 12 Juillet } 2007 \text { au } 17 \text { Janvier } \\
\text { 2010) }\end{array}$ & $1 / 3=0.33$ \\
\hline $\begin{array}{c}\text { L. Abaga Nchama } \\
\text { (du } 17 \text { Janvier } 2010 \text { à Décembre } \\
\text { 2016) }\end{array}$ & $1 / 6=0.16$ \\
\hline Taux de rotation pour la période & $\begin{array}{l}\text { Nombre de changements total / durée de la période } \\
\qquad 3 / 23=0.1304\end{array}$ \\
\hline
\end{tabular}

Source : Construction des auteurs d'après Cukierman et al. (1992).

Annexe $2:$ Tests de stationnarité des variables

\begin{tabular}{|c|c|c|c|c|c|c|c|c|c|}
\hline \multirow{3}{*}{ VARIABLES } & \multicolumn{4}{|c|}{ TEST DE IPS } & \multicolumn{4}{|c|}{ TEST LLC } & \multirow{3}{*}{$\begin{array}{c}\text { Ordre } \\
\text { d'intégration } \\
\text { / Décision }\end{array}$} \\
\hline & \multicolumn{2}{|c|}{ En niveau } & \multicolumn{2}{|c|}{$\begin{array}{c}\text { En difference } \\
1_{\text {ier }}\end{array}$} & \multicolumn{2}{|c|}{ En niveau } & \multicolumn{2}{|c|}{$\begin{array}{c}\text { En difference } \\
1_{\text {ier }}\end{array}$} & \\
\hline & T-bar & $\begin{array}{c}\text { P- } \\
\text { Value }\end{array}$ & T-bar & $\begin{array}{c}\mathrm{P}- \\
\text { value }\end{array}$ & LLcal & $\begin{array}{c}\mathrm{P}- \\
\text { value }\end{array}$ & LLcal & $\begin{array}{c}\mathrm{P}- \\
\text { value }\end{array}$ & \\
\hline $\log _{-}$Tinflatrans $\mathrm{i}_{\mathrm{i}, \mathrm{t}}$ & $\begin{array}{c}- \\
5.0217 \\
\end{array}$ & 0.0000 & - & - & $\begin{array}{c}- \\
4.7617 \\
\end{array}$ & 0.0000 & - & - & $\mathrm{I}(0)^{* * *}$ \\
\hline Logt_Pib ${ }_{i, t}$ & $\begin{array}{c}- \\
4.4969\end{array}$ & 0.0000 & - & - & $\begin{array}{c}- \\
2.9981\end{array}$ & 0.0014 & - & - & $\mathrm{I}(0) * * *$ \\
\hline $\log _{\text {Lebu }} \mathrm{i}_{\mathrm{i}, \mathrm{t}}$ & $\begin{array}{c}- \\
3.4539\end{array}$ & 0.0003 & - & - & $2 . \overline{-}$ & 0.0170 & - & - & $\begin{array}{c}\mathrm{I}(0)^{* * *} / \mathrm{I} \\
(0)^{* *}\end{array}$ \\
\hline 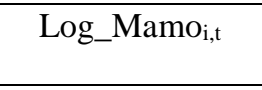 & 3.3222 & 0.9996 & $\begin{array}{c}- \\
5.6278 \\
\end{array}$ & 0.0000 & 2.9671 & 0.9985 & $\begin{array}{c}- \\
5.9966 \\
\end{array}$ & 0.0000 & $\mathrm{I}(1)^{* * *}$ \\
\hline $\log _{-} \operatorname{Ouv}_{\mathrm{i}, \mathrm{t}}$ & $\begin{array}{c}- \\
1.7394 \\
\end{array}$ & 0.0410 & - & - & $\begin{array}{c}- \\
1.0627 \\
\end{array}$ & 0.1440 & $\begin{array}{c}- \\
7.2974 \\
\end{array}$ & 0.0000 & $\begin{array}{c}\mathrm{I}(0)^{* * / \mathrm{I}} \\
(1)^{* * *}\end{array}$ \\
\hline $\log \_$Txurb $_{i, t}$ & $\begin{array}{c}- \\
4.0067 \\
\end{array}$ & 0.0000 & - & - & $\begin{array}{c}- \\
3.4755 \\
\end{array}$ & 0.0003 & - & - & $\mathrm{I}(0) * * *$ \\
\hline $\log _{-}$Agri $_{i, t}$ & $\begin{array}{c}- \\
2.4566 \\
\end{array}$ & 0.0070 & - & - & $\begin{array}{c}- \\
2.7848 \\
\end{array}$ & 0.0027 & - & - & $\mathrm{I}(0)^{* * *}$ \\
\hline $\log _{-}$Tor $_{\mathrm{i}, \mathrm{t}}$ & 2.5234 & 0.9942 & $\begin{array}{c}- \\
5.8060\end{array}$ & 0.0000 & 0.8575 & 0.8044 & $\begin{array}{c}- \\
4.8459 \\
\end{array}$ & 0.0000 & $\mathrm{I}(1)^{* * *}$ \\
\hline $\log _{-} \operatorname{Libc}_{i, t}$ & 2.5234 & 0.9942 & $\begin{array}{c}- \\
5.8060 \\
\end{array}$ & 0.0000 & 0.8575 & 0.8044 & $\begin{array}{c}- \\
4.8459 \\
\end{array}$ & 0.0000 & $\mathrm{I}(1)^{* * *}$ \\
\hline
\end{tabular}

Source : Calculs des auteurs à partir de Stata 13 . 
Avec : I(0) : Série stationnaire en niveau ou intégrée d'ordre $0 ; \mathbf{I}(\mathbf{1})$ : Série stationnaire en différence première ou au niveau du trend ou encore intégré d'ordre 1 ; IPS : Im-pesaran et Shin ; LLC : Lin Levine et Chu ; $\boldsymbol{\alpha}=$ $1 \% * * * ; \boldsymbol{\alpha}=5 \% * *$ et $\boldsymbol{\alpha}=10 \% *$ le degré de significativité.

\section{Annexe 3 : Construction de l'indice d'indépendance légal (LIBC-CWN)} de la BEAC conformément aux statuts de 2010

Note : Les données de base sur les $\mathbf{1 6}$ mesures initiales ont été regroupées en 8 critères comme suit :

1. Les quatre variables concernant la nomination et la durée du mandat du gouverneur de la banque centrale ont été regroupées en une seule variable égale à la moyenne des quatre composantes c'est-à-dire $(\mathbf{1 a + 1 b + 1 c + 1 d ) / 4 . ~}$

2. Les trois variables de formulation de la politique $\mathbf{2 a}, \mathbf{2 b}$ et $\mathbf{2 c}$ ont été regroupées en une seule variable en calculant une moyenne pondérée respectivement avec les poids $\mathbf{0 . 5}, \mathbf{0 . 2 5}, \mathbf{0 . 2 5}$ ).

3. La variable concernant les objectifs a été traitée séparément.

4. Le critère Avance concernant les limites sur les prêts.

5. Prêts sécurisés dans le cadre des limites sur les prêts.

6. Le critère des conditions des prêts dans le cadre des limites sur les prêts.

7. Les critères des emprunteurs potentiels de la banque en vertu des limites sur les prêts.

8. Les quatres dernières variables du groupe ont été regroupées en une seule variable en utilisant des poids égaux à savoir $(\mathbf{4 e}+\mathbf{4 f}+\mathbf{4} g+\mathbf{4 h}) / \mathbf{4}$. 


\begin{tabular}{|c|c|c|c|c|c|}
\hline Caractéristiques & \begin{tabular}{|c|}
$\begin{array}{c}\text { Code } \\
\text { variable }\end{array}$ \\
\end{tabular} & Poids & Score & $\begin{array}{l}\text { Score } \\
\text { choisi }\end{array}$ & \begin{tabular}{|l}
$\begin{array}{l}\text { Références } \\
\text { statutaires }\end{array}$ \\
\end{tabular} \\
\hline 1/Indépendance des dirigeants & ID & 0.20 & & & \\
\hline \begin{tabular}{|l} 
a) Durée du mandat \\
- $\quad 28 a$. \\
- 8 ans. \\
- 4 ans. \\
- $\quad 4$ ans. \\
ans ou à la discrétion du responsable de la nomination.
\end{tabular} & DM & 0.05 & $\begin{array}{l}1.00 \\
0.75 \\
0.50 \\
0.25 \\
0.00\end{array}$ & 0.75 & Art. 50. \\
\hline \begin{tabular}{|l} 
b) Procédure de nomination du gouverneur \\
- Conseil d'administration de la Banque Centrale (BC). \\
- Décision collective de la BC, des pouvoirs exécutif et législatif. \\
- Pouvoir législatif. \\
- Pouvoir exécutif collectivement (ex. : conseil des ministres). \\
- Un seul ou deux représentants de l'exécutif.
\end{tabular} & PNG & 0.05 & $\begin{array}{l}1.00 \\
0.75 \\
0.50 \\
0.25 \\
0.00\end{array}$ & 0.75 & Art. 50. \\
\hline $\begin{array}{l}\text { c) Conditions de limogeage du gouverneur ou des membres du conseil } \\
\text { - Non-prévu par la loi. } \\
\text { - Pour raisons non-liées à la politique monétaire. } \\
\text { - A la discrétion du conseil d'administration de la BC. } \\
\text { - A la discrétion du pouvoir législatif pour motif lié à la politique } \\
\text { - monétaire. } \\
\text { - A la discrétion du pouvoir législatif sans condition de motif. } \\
\text { - } \text { monétaire. } \\
\text { - A la discrétion du pouvoir exécutif sans condition de motif. }\end{array}$ & CLG & 0.05 & $\begin{array}{l}1.00 \\
0.83 \\
0.67 \\
0.50 \\
\\
0.33 \\
0.17 \\
0.00\end{array}$ & 0.83 & Art. 53. \\
\hline $\begin{array}{l}\text { d) Le gouverneur ou les membres du conseil peuvent-ils exercer d'autres } \\
\text { fonctions ? } \\
\text { - Non } \\
\text { - Seulement avec préalable autorisation du pouvoir exécutif. } \\
\text { - Aucune réglementation en la matière. }\end{array}$ & GMF & 0.05 & $\begin{array}{l}1.00 \\
0.50 \\
0.00\end{array}$ & 1.00 & Art. 57. \\
\hline $2 /$ Formulation de la politique monétaire & FPM & 0.15 & & & \\
\hline $\begin{array}{l}\text { a) Qui formule le politique monétaire? } \\
\text { - La Banque seule. } \\
\text { - La Banque participe mais, a peu de pouvoir. } \\
\text { - La Banque est un conseiller du gouvernement. } \\
\text { - La Banque n'a aucun pouvoir. }\end{array}$ & QPM & 0.05 & $\begin{array}{l}1.00 \\
0.67 \\
0.33 \\
0.00\end{array}$ & 1.00 & Art. 1. \\
\hline $\begin{array}{l}\text { b) Poids de la Banque Centrale dans la résolution des conflits avec le } \\
\text { gouvernement } \\
\text { - La Banque a le dernier mot concernant ses objectifs tels que définis } \\
\text { par la loi. } \\
\text { - Le gouvernement tranche pour tout ce qui ne touche pas aux } \\
\text { objectifs statutaires de la BC, ou en cas de conflit interne au sein } \\
\text { de la BC. } \\
\text { - Un conseil triparti (BC, pouvoirs exécutif et législatif) règle les } \\
\text { conflits. } \\
\text { - Le pouvoir législatif a le dernier mot en ce qui concerne les conflits } \\
\text { liés à la politique monétaire. } \\
\text { Le pouvoir exécutif a le dernier mot pour les conflits sur la } \\
\text { politique monétaire mais, selon un processus prévu et avec } \\
\text { possible protestation de la BC. } \\
\text { Le pouvoir exécutif décide sans condition. }\end{array}$ & PBRCG & 0.05 & $\begin{array}{l}1.00 \\
0.80 \\
0.60 \\
\\
0.40 \\
0.20 \\
0.00\end{array}$ & 0.80 & Art. 6 et 7 . \\
\hline $\begin{array}{l}\text { a) Participation de la Banque Centrale au processus budgétaire de l'Etat } \\
\text { - La Banque Centrale joue un rôle actif. } \\
\text { - La Banque Centrale n'intervient pas. }\end{array}$ & PBCPBE & 0.05 & $\begin{array}{l}1.00 \\
0.00\end{array}$ & 1.00 & $\begin{array}{c}\text { Art. } 12 \text { et } \\
21 .\end{array}$ \\
\hline
\end{tabular}




\begin{tabular}{|c|c|c|c|c|c|}
\hline 3/ Objectifs attribués à la Banque Centrale & OBC & 0.15 & & & \\
\hline $\begin{array}{l}\text { - La stabilité des prix mentionnée comme objectif unique ou } \\
\text { prioritaire. La BC ayant le dernier mot en cas de conflit d'objectif } \\
\text { (autres objectif : plein emploi, etc...). } \\
\text { - La stabilité des prix est l'unique objectif. } \\
\text { - La stabilité de la valeur de la monnaie est mentionnée parmi d'autres } \\
\text { objectifs tels que solidité du système bancaire. } \\
\text { - La stabilité de la valeur de la monnaie est associée à d'autres objectifs } \\
\text { - } \quad \text { qui lui sont incompatibles. } \\
\text { - } \quad \text { Objectifs autres que la stabilité des prix. }\end{array}$ & & & $\begin{array}{l}1.00 \\
0.80 \\
0.60 \\
\\
0.40 \\
0.20 \\
0.00\end{array}$ & 0.60 & Art. 1. \\
\hline 4/Limitations des crédits au gouvernement & LCG & 0.50 & & & \\
\hline $\begin{array}{l}\text { a) Les avances monétaires au gouvernement } \\
\text { - Aucune avance n'est autorisée. } \\
\text { - Avances permises mais dans des limites strictes (ex : maximum des } \\
\text { avances }=15 \% \text { des revenus du gouvernement). } \\
\text { - Avance permises avec les limites plutôt lâches (ex : maximum des } \\
\text { avances }=\text { au-delà de } 15 \% \text { des revenus du gouvernement). } \\
\text { - Pas de limites légales. }\end{array}$ & AMG & 0.15 & $\begin{array}{l}1.00 \\
0.67 \\
0.33 \\
0.00\end{array}$ & 0.67 & $\begin{array}{c}\text { Art. } 17 \text { et } \\
18 .\end{array}$ \\
\hline 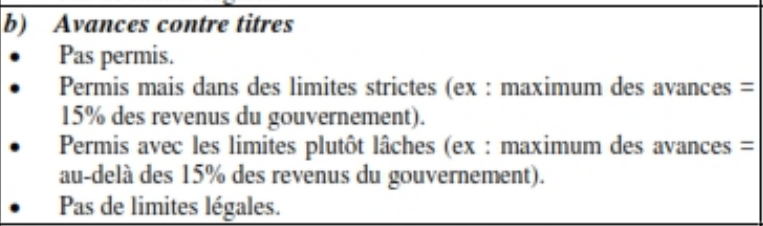 & ACT & 0.10 & $\begin{array}{l}1.00 \\
0.67 \\
0.33 \\
0.00\end{array}$ & 0.33 & $\begin{array}{c}\text { Art. } 15 \text { et } \\
16 .\end{array}$ \\
\hline 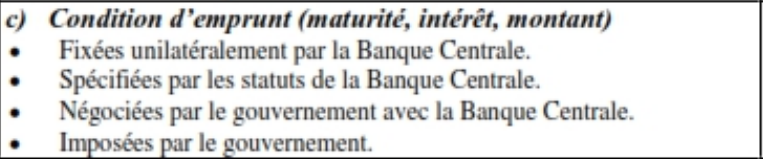 & $\mathrm{CE}$ & 0.10 & $\begin{array}{l}1.00 \\
0.67 \\
0.33 \\
0.00\end{array}$ & 0.33 & Art. 16. \\
\hline 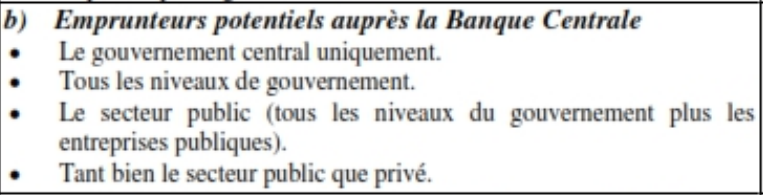 & EPBC & 0.05 & $\begin{array}{l}1.00 \\
0.67 \\
0.33 \\
0.00\end{array}$ & 0.00 & Art. 17. \\
\hline $\begin{array}{l}\text { e) Les limites de concours de la Banque Centrale au } \\
\text { gouvernement définies comme: } \\
\text { - Montant d'argent. } \\
\text { - Fraction du capital ou du passif de la Banque Centrale. } \\
\text { - Fraction du revenu du gouvernement. } \\
\text { Fraction des dépenses du gouvernement. }\end{array}$ & LCBCG & 0.025 & $\begin{array}{l}1.00 \\
0.67 \\
0.33 \\
0.00\end{array}$ & 0.33 & Art. 11. \\
\hline $\begin{array}{ll}f & \text { Maturité des prêts : } \\
& <6 \text { mois. } \\
\text { - } & <\text { lan. } \\
\text { - } & <\text { lan. } \\
\text { - Non-défini dans la loi. }\end{array}$ & MP & 0.025 & $\begin{array}{l}1.00 \\
0.67 \\
0.33 \\
0.00\end{array}$ & 0.00 & Art. 11. \\
\hline $\begin{array}{l}\text { g) Les taux intérêts sur les avances devraient être: } \\
\text { - > aux taux minima. } \\
\text { - } \quad \text { taux de marché. } \\
\text { - Aucun taux maxima. } \\
\text { - Pas d'intérêt perçu de taux les avances au gouvernement. }\end{array}$ & TIA & 0.025 & $\begin{array}{l}1.00 \\
0.75 \\
0.50 \\
0.25 \\
0.00\end{array}$ & 0.25 & Art. 11. \\
\hline $\begin{array}{l}\text { h) La Banque Centrale peut-elle autorisée à acheter ou vendre les titres } \\
\text { publics sur le marché primaire? } \\
\text { - Oui. } \\
\text { - Non. }\end{array}$ & BCAVTP & 0.025 & $\begin{array}{l}1.00 \\
0.00\end{array}$ & 0.00 & $\begin{array}{c}\text { Art. } 15 \text { et } \\
16 .\end{array}$ \\
\hline
\end{tabular}

